\title{
On asymptotically flat solutions of Einstein's equations periodic in time I. Vacuum and electrovacuum solutions
}

\author{
J Bičák ${ }^{1,3}$, M Scholtz ${ }^{1,3}, \mathbf{P}$ Tod $^{2,1}$ \\ ${ }^{1}$ Institute of Theoretical Physics, Faculty of Mathematics and Physics, \\ Charles University, V Holešovičkách 2, 18000 Prague 8, Czech Republic \\ 2 Mathematical Institute, Oxford OX1 3LB, UK \\ ${ }^{3}$ Max Planck Institute for Gravitational Physics, Albert Einstein Institute,
Am Mühlenberg 1, 14476 Golm, Germany
E-mail: bicak@mbox.troja.mff.cuni.cz, scholtzzz@gmail.com,
paul.tod@sjc.ox.ac.uk
}

Abstract. By an argument similar to that of Gibbons and Stewart [10], but in a different coordinate system and less restrictive gauge, we show that any weaklyasymptotically-simple, analytic vacuum or electrovacuum solutions of the Einstein equations which are periodic in time are necessarily stationary.

\section{Introduction}

The inspiral and coalescence of binary black holes or neutron stars appears to be the most promising source for the detectors of gravitational waves, so that there has been much effort going into the development of numerical codes and analytic approximation methods to find the corresponding solutions of Einstein's equations. One of the recent approaches assumes the existence of a helical Killing vector $k$ (see e.g. [25]). The field is assumed stationary in a rotating frame where $k$ generates time translations but $k$ becomes null at the light cylinder and is spacelike outside. $k$ has the form $k=\partial_{t}+\omega \partial_{\phi}$ where $\partial_{t}$ is timelike and $\partial_{\phi}$ is spacelike with circular orbits with parameter length $2 \pi$ (except where $\partial_{\phi}=0$ ); $\omega=$ constant. The space-time is not stationary but it is still periodic where $k$ is spacelike. Requiring the helical symmetry for a binary system implies equal amounts of outgoing and incoming radiation so that the space-time, containing energy radiated all times is not expected to be asymptotically flat. A corresponding solution in Maxwell's theory for two opposite point charges moving on circular orbits was considered a long time ago by Schild [19]. The properties of the field were analyzed recently in the Newman-Penrose formalism in [1]. The rather complicated periodicity properties of the solution became apparent as well as 
its asymptotic behaviour: at $\mathcal{I}^{-}$the advanced fields exhibit the standard Bondi-type expansion and peeling, whereas the retarded fields do decay with $r \rightarrow \infty$ but in an oscillatory manner like $(\sin r) / r$. Hence for the retarded plus advanced solution no radiation field is asymptotically defined. Naturally, one would like to go beyond the linearized theory. There are special exact, time-dependent, solutions known, for example, Szekeres's dust solution, which has in general no Killing vector, which can be matched to an exterior Schwarzschild metric [3]. One can construct oscillating spherical shells of dust particles moving with the same angular momentum, but in every tangential direction, or oscillating Einstein clusters which are matched to the Schwarzschild space-time outside [8]. Can there be periodic solutions representing "bound states" of gravitational or electromagnetic waves so that the radiation field at infinity vanishes and the Bondi mass remains constant?

There have been various attempts to prove that, while solutions of the vacuum Einstein equations can be genuinely periodic in a suitable time-coordinate (so not time-independent), these solutions cannot be asymptotically flat. These started with [15] and [16], with a summary in English in [17], and [24] and more recently was considered in [10]. The method in [15] considers vacuum metrics which are everywhere nonsingular, weak and asymptotically-flat and which can be expanded in a series in some parameter, with the flat metric as the first term in the series. Each term in the series is assumed to be periodic in a fixed Minkowski time-coordinate and to satisfy the de Donder gauge condition. The second and third terms, call them $v_{a b}$ and $w_{a b}$ respectively, are expanded as Fourier series in the background time-coordinate and the Einstein equations then imply that $v_{a b}$ satisfies the source-free wave equation, and $w_{a b}$ satisfies a wave equation whose source is a quadratic expression in $v_{a b}$. Assuming that the solution for $v_{a b}$ is everywhere regular, the author shows that there cannot be an asymptotically-flat solution for $w_{a b}$ unless $v_{a b}$ vanishes. Therefore the spacetime is flat. In [16], a similar calculation when $v_{a b}$ is regular only outside a certain radius leads to the conclusion that $v_{a b}$ must be time-independent in order to have asymptotically-flat $w_{a b}$, and the space-time is stationary. In [24] it was observed by integrating the Einstein pseudotensor and matter energy-momentum tensor over a 4-dimensional volume that "the mean value of power radiated by a periodic, asymptotically Minkowskian gravitational field is equal to zero". The question of existence of periodic fields was left open. In [10] the authors used the spin-coefficient formalism (see e.g. [14], [22]) to study the system of conformal Einstein equations of Friedrich [5]. A coordinate system is based on two families of null hypersurfaces, incoming from past null infinity $\mathcal{I}^{-}$and labelled by constant $v$ and outgoing near $\mathcal{I}^{-}$ and labeled by constant $u$. The authors define periodicity as meaning periodic in $v$ in these coordinates and are then able to prove that, at $\mathcal{I}^{-}$, the $u$-derivatives of all orders of all components of the metric are independent of $v$. They conclude that if the metric is analytic in these coordinates, then it necessarily has a Killing vector, which in these coordinates is $\partial_{v}$, at least in a neighbourhood of $\mathcal{I}^{-}$. Thus any analytic metric, periodic in their sense, has such a Killing vector. While certainly correct, there is a problem with this conclusion in that, by construction, the Killing vector is null wherever it is defined, and reduces at $\mathcal{I}^{-}$to a constant translation along the generators. These are strong conditions and in fact no Killing vector in flat space has these properties (any null Killing vector is necessarily a null translation, and a null translation is zero along one generator of $\mathcal{I}) \ddagger$. Thus flat space is not periodic according

$\ddagger$ For example the null translation $\partial_{t}+\partial_{z}$ becomes $2 \cos ^{2}(\theta / 2) \partial_{v}$ on $\mathcal{I}^{-}$, which vanishes at $\theta=\pi$. 
to the definition of [10] and nor is any of the familiar stationary, asymptotically flat solutions, for example the Schwarzschild solution, so that this definition of periodicity is 'too strong'.

For convenience, we follow [10] in working at $\mathcal{I}^{-}$rather than $\mathcal{I}^{+}$, though this is trivial to switch, but we shall make a weaker definition of 'periodic in time' which will permit metrics stationary near $\mathcal{I}^{-}$and indeed will allow only these for analytic, asymptotically-flat vacuum or electrovac metrics. We follow the method of [10] for both the vacuum and electrovac field equations, deferring other cases to a second article, but in a different coordinate and tetrad system. Our coordinate and tetrad system is similar to the one used at $\mathcal{I}$ in [14], and to prove the existence of a symmetry at the event horizon in [11] and at a compact Cauchy horizon in [12]. We also differ from [10] in the choice of conformal gauge. In [10] the unphysical Ricci scalar is set to zero by a choice of conformal factor obtained by solving a wave equation. However, the solution of the characteristic IVP for this wave equation as posed in [10] will not in general be periodic, so that the rescaled, unphysical metric would not in general share the periodicity of the physical metric - in fact, in the particular case of the Reissner-Nordström solution this gauge choice is compatible with periodicity only for zero mass, as we show in Appendix C. Thus we assume that there is at least one conformal factor which is periodic and then modify this choice in the course of the calculation in order to simplify the spin coefficients. From this point on, our method is then essentially the same as in [10], though a little more complicated, and we arrive at the same conclusion, but now with a Killing vector which is time-like in the interior, at least near to $\mathcal{I}^{-}$. The condition of time-like periodicity which we impose is as follows: a space-time is time-like periodic if there is a discrete isometry taking any point of the physical space-time to a point in its chronological future. To define timelike periodicity at $\mathcal{I}^{-}$for an asymptotically-flat space-time, we require this isometry to extend to an isometry of a neighbourhood of $\mathcal{I}^{-}$which preserves the generators of $\mathcal{I}^{-}$. In particular, we require the existence of at least one $\Omega$ which conformally compactifies the space-time and preserves the periodicity. The isometry has to be a supertranslation [22],

$$
v \rightarrow v+a(\theta, \phi),
$$

on $\mathcal{I}^{-}$, in terms of the usual coordinates $(v, \theta, \phi)$ on $\mathcal{I}^{-}$and we shall assume that $a \neq 0$. (We could imagine allowing $a$ to vanish on some generators of $\mathcal{I}^{-}$, since as noted above periodicity along a null translation in flat space would appear like this at $\mathcal{I}^{-}$, but this would be null-periodicity rather than time-like periodicity.) We could assume further that $a$ is actually a positive constant but this turns out not to be necessary, as we shall find that, for analytic space-times, this assumption of periodicity necessarily leads to a space-time metric with a Killing vector which, in coordinates to be defined, is $\partial_{v}$ and is time-like near $\mathcal{I}^{-}$. Our result is

Theorem 1.1. A weakly-asymptotically simple, vacuum or electrovac, time-periodic space-time which is analytic in a neighbourhood of $\mathcal{I}^{-}$in the coordinates introduced below necessarily has a Killing vector which is time-like in the interior and extends to a translation on $\mathcal{I}^{-}$.

Thus there are no non-trivial time-periodic solutions satisfying these conditions, in the sense that they would necessarily be actually time-independent if time-periodic. In a later article, we shall prove the corresponding result for the Einstein equations coupled to either a massless scalar field with the usual energy-momentum tensor, or a 
solution of the conformally-invariant wave equation with the energy-momentum tensor from p125 of [18] (sometimes called the 'new improved energy momentum tensor').

The method of proof requires the assumption of analyticity. It was shown in [6] that there are vacuum solutions analytic near $\mathcal{I}^{-}$. However, one would like either to drop the assumption of analyticity, for example following the lead of [7] or [9] with a similar problem, or to prove that it follows from the assumptions of periodicity and asymptotic-flatness. It remains to be seen in what circumstances this can be done since, as noted above, there are non-analytic solutions with matter in periodic motion and matched to a (static) Schwarzschild exterior.

While this work is primarily motivated by an interest in the possibility or impossibility of helical motions, it is worth noting the connection with the question of the inheritance of symmetry. Recall that, for a solution of Einstein's field equations with matter, the matter is said to inherit the symmetry of the metric if any isometry of the metric is necessarily a symmetry of the matter. There are explicit solutions of the Einstein-Maxwell equations known for which an isometry of the metric is not a symmetry of the Maxwell field [13] but these solutions are not asymptotically-flat. In [21] some other references may be found for explicit solutions with Maxwell fields which do not share the symmetry of the metric. The same will be true for some Robinson-Trautman solutions with null electromagnetic field which may depend on time though the metric is static (see [21], §28.2) These solutions will very likely have wire singularities extending to infinity. From Theorem 1.1 noninheritance cannot happen with asymptotically-flat, analytic solutions:

Corollary 1.2. In any weakly-asymptotically simple, stationary electrovac space-time which is analytic in a neighbourhood of $\mathcal{I}^{-}$in the coordinates introduced below, the Maxwell field is also stationary.

One can raise the question of inheritance also for Einstein-scalar field solutions but the answer is rather different: for a massive (complex) Klein-Gordon field there do exist solutions, the so-called 'boson stars', for which the metric is spherically-symmetric, asymptotically-flat and static but the scalar field has a phase linear in time (see e.g. [2]); however these solutions are not analytic at infinity and, by a scaling argument, such solutions do not exist with massless scalar fields. In a later article, we shall obtain this result as a corollary of the result corresponding to Theorem 1.1. In that subsequent work we start from the conformal Einstein field equations with a general energy-momentum tensor as a source and specialize them to scalar field cases.

In Section 2 we analyze the conformal Einstein-Maxwell equations. We first rewrite Maxwell's equations in the unphysical space-time, then translate the physical Bianchi identities and obtain differential equations for the unphysical Weyl spinor and Ricci spinor. In Appendix A we summarize a number of quantities, their relations and behaviour under conformal transformations in the Newman-Penrose formalism ([14]); these are extensively used in the main text and in Appendices B and C. In particular, all conformal equations for the gravitational and electromagnetic field analyzed in terms of spinors in Section 2 are projected on the spin basis (i.e. the null tetrad) and written down in the Newman-Penrose formalism in Appendix B. In Section 3 a suitable coordinate system and a convenient Newman-Penrose null tetrad which gives special values to some of the Newman-Penrose spin-coefficients are introduced in the neighbourhood of $\mathcal{I}^{-}$. As noted above, these differ from those used by the authors of [10], and we shall show the differences explicitly in Section 3. In Appendix C we demonstrate that in contrast to [10] our choice of gauge admits simple static (i.e. 
'periodic') space-times like flat space and the Reissner-Nordström metric. In Section 4 we follow [10] (although in a different conformal gauge) and study the problem in the NP formalism in the unphysical space-time, with data on $\mathcal{I}^{-}$. Assuming periodicity along $\mathcal{I}^{-}$we first discover that the only possibility is the independence of all geometric quantities of an affine parameter $v$ along $\mathcal{I}^{-}$. By induction we then prove that all derivatives of all geometric quantities, including the physical metric components, in the direction into the physical space-time must also be $v$-independent. The proof of Theorem 1.1 and Corollary 1.2 then follows from the assumed analyticity.

This paper arose from a collaboration after P. T. posted his work [23] on the gr-qc arXive and J. B. informed him that he and his PhD. student M. S. were already engaged in tackling the same problem [20].

\section{The conformal Einstein-Maxwell equations}

We first introduce conformal equations for the gravitational and electromagnetic field in the formalism of 2-component spinors. In Appendix B these equations are written down explicitly after the projection on a spin basis, in the form employed in the Newman-Penrose formalism. In the physical space-time, Maxwell's equations without sources are simply§ (see e.g. [22])

$$
\tilde{\nabla}^{A A^{\prime}} \tilde{\phi}_{A B}=0 \text {. }
$$

They are conformally invariant if under conformal rescaling the Maxwell spinor $\phi_{A B}$ tranforms with conformal weight 1 ,

$$
\tilde{\phi}_{A B}=\Omega \phi_{A B},
$$

when the convention used in this article for conformal rescaling is $\tilde{\epsilon}_{A B}=\Omega^{-1} \epsilon_{A B}$.

From the transformation of the derivative operator (see (A12)), in the unphysical space-time equations (2) become

$$
\nabla^{A A^{\prime}} \phi_{A B}=0 \text {. }
$$

The situation is more complicated in the case of the gravitational field. The physical Bianchi identities read

$$
\tilde{\nabla}_{C^{\prime}}^{D} \tilde{\Psi}_{A B C D}=\tilde{\nabla}_{(C}^{D^{\prime}} \tilde{\Phi}_{A B) C^{\prime} D^{\prime}}
$$

where $\tilde{\Psi}_{A B C D}$ and $\tilde{\Phi}_{A B C^{\prime} D^{\prime}}$ are the Weyl and the Ricci spinor, respectively. Using the rules for the conformal transformation of these spinors (eq. (A15) and (A17)) we find

$$
\Omega^{2} \nabla_{C^{\prime}}^{D} \psi_{A B C D}=\Omega \nabla_{(C}^{D^{\prime}} \Phi_{A B) C^{\prime} D^{\prime}}+\left(\nabla_{\left(A^{D^{\prime}} \Omega\right.}^{D^{\prime}}\right) \Phi_{B C) C^{\prime} D^{\prime}}+\nabla_{(C}^{D^{\prime}} \nabla_{A\left(C^{\prime}\right.} \nabla_{\left.\left.D^{\prime}\right) B\right)} \Omega
$$

where $\psi_{A B C D}=\Omega^{-1} \Psi_{A B C D}$. These equations are the physical Bianchi identities written in terms of the quantities in the unphysical space-time. We simplify them by employing Einstein's equations in the physical space-time,

$$
\tilde{\Phi}_{A B A^{\prime} B^{\prime}}=k \tilde{\phi}_{A B} \overline{\tilde{\phi}}_{A^{\prime} B^{\prime}} \text {. }
$$

Here we used the fact that the physical scalar curvature vanishes for the electromagnetic field; we put the constant factor $k$ on the r.h.s. of (7) equal to 1 
following the convention of [14], unlike, e.g. [18]. From eqs. (A15), (7) and (3) we obtain

$$
\nabla_{A\left(A^{\prime}\right.} \nabla_{\left.B^{\prime}\right) B} \Omega=\Omega^{3} \phi_{A B} \bar{\phi}_{A^{\prime} B^{\prime}}-\Omega \Phi_{A B A^{\prime} B^{\prime}} .
$$

Applying $\nabla_{C}^{D^{\prime}}$, symmetrizing and using Maxwell's equations (4), we can express the term containing the third derivative of $\Omega$ appearing in (6) as follows:

$$
\begin{aligned}
& \nabla_{(C}^{D^{\prime}} \nabla_{A\left(C^{\prime}\right.} \nabla_{\left.\left.D^{\prime}\right) B\right)} \Omega= \\
& 3 \Omega^{2} \bar{\phi}_{C^{\prime} D^{\prime}} \phi_{(A B} \nabla_{C)}^{D^{\prime}} \Omega+\Omega^{3} \bar{\phi}_{C^{\prime} D^{\prime}} \nabla_{(C}^{D^{\prime}} \phi_{A B)}-\Omega \nabla_{(C}^{D^{\prime}} \Phi_{A B) C^{\prime} D^{\prime}}-\left(\nabla_{\left(C^{\prime}\right.}^{D^{\prime}} \Omega\right) \Phi_{A B) C^{\prime} D^{\prime}} .
\end{aligned}
$$

Inserting this result into (6) we arrive at the conformal Bianchi identities for the Einstein-Maxwell field expressed in terms of the quantities in the unphysical spacetime:

$$
\nabla_{A^{\prime}}^{D} \psi_{A B C D}=3 \bar{\phi}_{A^{\prime} B^{\prime}} \phi_{(A B} \nabla_{C)}^{B^{\prime}} \Omega+\Omega \bar{\phi}_{A^{\prime} B^{\prime}} \nabla_{(C}^{B^{\prime}} \phi_{A B)} .
$$

Projecting these equations onto the spin basis we obtain the set of the equations which are explicitly written down (using the NP formalism) in Appendix B, see (B5a)-(B5h). Equations (9) are differential equations for the unphysical Weyl spinor. To obtain the equations for the Ricci spinor we use the Bianchi identities valid for quantities in the unphysical space-time:

$$
\nabla_{C^{\prime}}^{D} \Psi_{A B C D}=\nabla_{(C}^{D^{\prime}} \Phi_{A B) C^{\prime} D^{\prime}}
$$

Combining the last two equations, we get

$$
\nabla_{(C}^{B^{\prime}} \Phi_{A B) A^{\prime} B^{\prime}}=\psi_{A B C D} \nabla_{A^{\prime}}^{D} \Omega+3 \Omega \bar{\phi}_{A^{\prime} B^{\prime}} \phi_{(A B} \nabla_{C)}^{B^{\prime}} \Omega+\Omega^{2} \bar{\phi}_{A^{\prime} B^{\prime}} \nabla_{(C}^{B^{\prime}} \phi_{A B)} .
$$

In the following we shall also need the expression for quantities $\nabla_{A A^{\prime}} \nabla_{B B^{\prime}} \Omega$. Let us decompose $\nabla_{A A^{\prime}} \nabla_{B B^{\prime}} \Omega$ into its symmetric and antisymmetric parts,

$$
\nabla_{A A^{\prime}} \nabla_{B B^{\prime}} \Omega=\nabla_{A\left(A^{\prime}\right.} \nabla_{\left.B^{\prime}\right) B} \Omega+\frac{1}{2} \epsilon_{A^{\prime} B^{\prime}} \nabla_{A C^{\prime}} \nabla_{B}^{C^{\prime}} \Omega .
$$

The first term on the r.h.s. is given in (8), the second term can be decomposed again:

$$
\nabla_{A C^{\prime}} \nabla_{B}^{C^{\prime}} \Omega=\nabla_{C^{\prime}(A} \nabla_{B)}^{C^{\prime}} \Omega+\frac{1}{2} \epsilon_{A B} \square \Omega .
$$

Since the operator $\nabla_{C^{\prime}(A} \nabla_{B)}^{C^{\prime}}$ is just the commutator $\nabla_{[a} \nabla_{b]}$ contracted by $\epsilon^{A^{\prime} B^{\prime}}$, it annihilates scalar quantities. Using equations (8), (12) and (13) we obtain

$$
\nabla_{A A^{\prime}} \nabla_{B B^{\prime}} \Omega=\Omega^{3} \phi_{A B} \bar{\phi}_{A^{\prime} B^{\prime}}-\Omega \Phi_{A B A^{\prime} B^{\prime}}+\frac{1}{4} \epsilon_{A^{\prime} B^{\prime}} \epsilon_{A B} \square \Omega .
$$

It will be convenient to introduce the quantity

$$
F=\frac{1}{2} \Omega^{-1}\left(\nabla_{A A^{\prime}} \Omega\right)\left(\nabla^{A A^{\prime}} \Omega\right),
$$

which can be seen to be smooth in the unphysical space-time from the rule for the conformal transformation of the scalar curvature (A15) in the form

$$
\square \Omega=4 \Omega \Lambda-4 \Omega^{-1} \tilde{\Lambda}+4 F,
$$

since the physical scalar curvature $\tilde{\Lambda}=0$ for the electromagnetic field. From equation (14) we now obtain the following expressions for the second derivatives of $\Omega$ :

$\nabla_{A A^{\prime}} \nabla_{B B^{\prime}} \Omega=\Omega^{3} \phi_{A B} \bar{\phi}_{A^{\prime} B^{\prime}}-\Omega \Phi_{A B A^{\prime} B^{\prime}}+\epsilon_{A^{\prime} B^{\prime}} \epsilon_{A B}(F+\Omega \Lambda)$. 
Finally we wish to derive expressions for $\nabla_{A A^{\prime}} F$. Directly from the definition of the unphysical Riemann tensor and from the decomposition (A3), we have

$\left(\nabla_{A A^{\prime}} \nabla_{B B^{\prime}}-\nabla_{B B^{\prime}} \nabla_{A A^{\prime}}\right) \nabla^{B B^{\prime}} \Omega=-2 \Phi_{A B A^{\prime} B^{\prime}} \nabla^{B B^{\prime}} \Omega+6 \Lambda \nabla_{A A^{\prime}} \Omega$.

Employing Maxwell's equations (4) and the contracted Bianchi identities (A21), we find that equations (14) and (18) imply

$$
\nabla_{A A^{\prime}} F=\Omega^{2} \phi_{A}^{B} \bar{\phi}_{A^{\prime}}^{B^{\prime}} \nabla_{B B^{\prime}} \Omega-\Phi_{A B A^{\prime} B^{\prime}} \nabla^{B B^{\prime}} \Omega+\Lambda \nabla_{A A^{\prime}} \Omega .
$$

\section{Coordinates, tetrad and conformal gauge}

We assume that we have an analytic, time-periodic solution of the Einstein-Maxwell equations and an analytic, time-periodic conformal factor so that the unphysical metric with $\mathcal{I}^{-}$also has these properties. We construct a convenient coordinate system and a Newman-Penrose null tetrad in the neighborhood of $\mathcal{I}^{-}$(see Figs. 1, 2). We stay in the unphysical space-time in order to include $\mathcal{I}^{-}$. Let $\mathcal{S} \subset \mathcal{I}^{-}$be a particular spacelike 2 -sphere. We can introduce arbitrary coordinates $x^{I}, I=2,3$ on $\mathcal{S}$ and propagate them along $\mathcal{I}^{-}$by the condition

$$
\nabla_{\dot{\gamma}} x^{I}=0,
$$

where $\gamma=\gamma(v)$ is an affinely-parametrized null generator of $\mathcal{I}^{-}$. We may set $v=0$ on $\mathcal{S}$. The triple $\left(v, x^{2}, x^{3}\right)$ represents suitable coordinates on $\mathcal{I}^{-}$. In order to go into the interior of space-time we introduce the family of null hypersurfaces $\mathcal{N}_{v}$ orthogonal to $\mathcal{I}^{-}$and intersecting $\mathcal{I}^{-}$in the space-like cuts $\mathcal{S}_{v}$ of constant $v$. Let $\gamma^{\prime}=\gamma^{\prime}(r)$ be the null generators of the surface $\mathcal{N}_{v}$ labeled by $x^{I}$. Here, $r$ is the affine parameter which can be chosen so that $r=0$ on $\mathcal{I}^{-}$and $g(\mathrm{~d} v, \mathrm{~d} r)=1$ at $\mathcal{I}^{-}$. We propagate the coordinates $v$ and $x^{I}$ onto $\mathcal{N}_{v}$ by conditions

$$
\nabla_{\dot{\gamma}^{\prime}} x^{I}=0, \quad \nabla_{\dot{\gamma}^{\prime}} v=0 .
$$

We thus have established a coordinate chart

$$
x^{\mu}=\left(v, r, x^{2}, x^{3}\right), \quad \mu=0,1,2,3,
$$

in the neighbourhood of past null infinity. $\|$

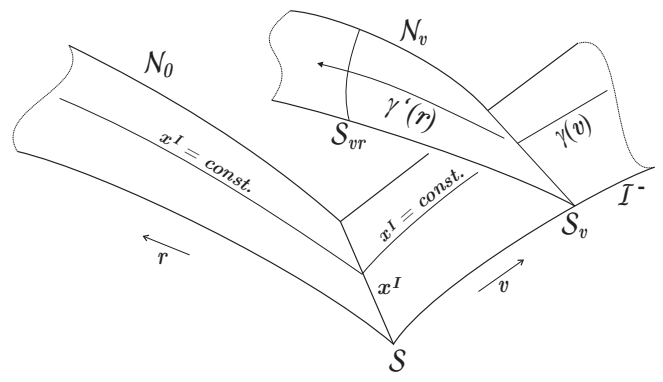

Figure 1. Construction of coordinate system.

|| Components of tensors with respect to the basis induced by these coordinates will be labelled by Greek letters $\mu, \nu, \ldots$. Components with respect to an arbitrary tetrad will be labelled by Latin letters $a, b, \ldots$ from the beginning of the alphabet. Indices labelled by capital letters $I, J, \ldots$ have values 2,3 . 
Next we construct a suitable Newman-Penrose null tetrad. $\mathcal{N}_{v}$ are null hypersurfaces $v=$ constant, therefore the gradient of $v$ is both tangent and normal to $\mathcal{N}_{v}$; we denote it by

$$
n_{a}=\nabla_{a} v .
$$

Since $n^{a}$ is tangent to $\gamma^{\prime}$ along which only $r$ varies,

$$
n=\frac{\partial}{\partial r} \text {. }
$$

On each cut $\mathcal{S}_{v r}: v, r=$ constant there exists exactly one null direction normal to $\mathcal{S}_{v r}$ not proportional to $n^{a}$. We choose the vector field $l^{a}$ to be parallel to this direction and normalize it by $n_{a} l^{a}=1$. It can be written in the form

$$
l=\frac{\partial}{\partial v}-H \frac{\partial}{\partial r}+C^{I} \frac{\partial}{\partial x^{I}} .
$$

On $\mathcal{I}^{-} l$ is tangent to the generators $\gamma(v)$, so functions $H$ and $C^{I}$ vanish on $\mathcal{I}^{-}$. The conformal gauge can be chosen so that

$$
\frac{\partial \Omega}{\partial r}=1 \quad \text { on } \mathcal{I}^{-} .
$$

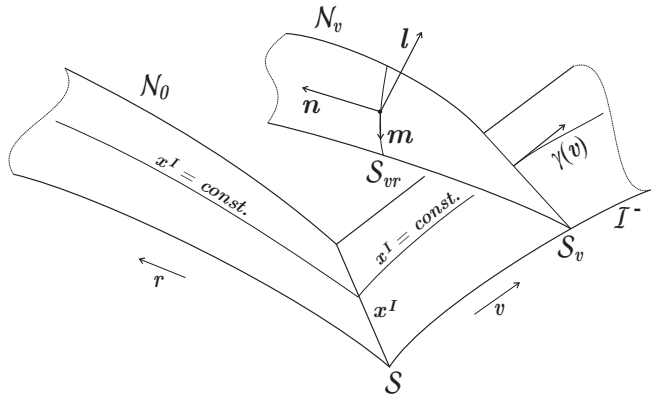

Figure 2. NP null tetrad.

Let us now turn to the 2 -spheres $\mathcal{S}_{v r}$ on which $\partial_{I}$ are basis vectors. Since $\mathcal{S}_{v r}$ is a space-like sphere, we choose, following standard procedure, a complex vector $m$ and its complex conjugate $\bar{m}$, such that

$$
m^{a} m_{a}=0, m^{a} \bar{m}_{a}=-1 ;
$$

$m$ has the form

$$
m=P^{I} \frac{\partial}{\partial x^{I}}
$$

where $P^{2}, P^{3}$ are complex functions. The coordinates $x^{I}$ can be chosen to be the standard spherical coordinates, $x^{I}=(\theta, \phi)$. Then the appropriate choice of the null vector $m$ at $\mathcal{I}^{-}$is (see e.g. [22])

$$
m=\frac{1}{\sqrt{2}}\left(\partial_{\theta}+\frac{i}{\sin \theta} \partial_{\phi}\right), P^{I}=\frac{1}{\sqrt{2}}\left(1, \frac{i}{\sin \theta}\right) .
$$

The vectors $m, \bar{m}$ are orthogonal to $l$ and $n$. The contravariant components of the tetrad read

$$
\begin{aligned}
& l^{\mu}=\left(1,-H, C^{2}, C^{3}\right), \\
& n^{\mu}=(0,1,0,0), \\
& m^{\mu}=\left(0,0, P^{2}, P^{3}\right) .
\end{aligned}
$$


The contravariant components of the metric tensor are given, regarding the relation $g^{\mu \nu}=2 l^{(\mu} n^{\nu)}-2 m^{(\mu} \bar{m}^{\nu)}$, by the matrix

$g^{\mu \nu}=\left(\begin{array}{cccc}0 & 1 & 0 & 0 \\ 1 & -2 H & C^{2} & C^{3} \\ 0 & C^{2} & -2 P^{2} \bar{P}^{2} & -P^{2} \bar{P}^{3}-P^{3} \bar{P}^{2} \\ 0 & C^{3} & -P^{2} \bar{P}^{3}-P^{3} \bar{P}^{2} & -2 P^{3} \bar{P}^{3}\end{array}\right)$.

Using (30) and the inverse of (31) we find the covariant components of the tetrad vectors:

$$
\begin{aligned}
l_{\mu} & =(H, 1,0,0), \\
n_{\mu} & =(1,0,0,0), \\
m_{\mu} & =\left(\omega, 0, R_{2}, R_{3}\right),
\end{aligned}
$$

where

$$
\begin{aligned}
& R_{2}=\frac{P^{3}}{P^{2} \bar{P}^{3}-P^{3} \bar{P}^{2}}, R_{3}=\frac{P^{2}}{P^{3} \bar{P}^{2}-P^{2} \bar{P}^{3}}, \\
& \omega=-C^{I} R_{I} .
\end{aligned}
$$

The covariant components of the metric are

$$
g_{\mu \nu}=\left(\begin{array}{cccc}
2 H-2 \omega \bar{\omega} & 1 & -\omega \bar{R}_{2}-\bar{\omega} R_{2} & -\omega \bar{R}_{3}-\bar{\omega} R_{3} \\
1 & 0 & 0 & 0 \\
-\omega \bar{R}_{2}-\bar{\omega} R_{2} & 0 & -2 R_{2} \bar{R}_{2} & -R_{3} \bar{R}_{2}-R_{2} \bar{R}_{3} \\
-\omega \bar{R}_{3}-\bar{\omega} R_{3} & 0 & -R_{3} \bar{R}_{2}-R_{2} \bar{R}_{3} & -2 R_{3} \bar{R}_{3}
\end{array}\right) .
$$

The vectors $l, n, m$ and $\bar{m}$ constitute the NP tetrad. However, it is not unique since there is a rotation gauge freedom $m \rightarrow e^{i \chi} m$ which will be used later. Following the standard notation of the NP formalism (e.g. [14], [22]), we define the operators:

$$
D=l^{a} \nabla_{a}, \quad \Delta=n^{a} \nabla_{a}, \quad \delta=m^{a} \nabla_{a} .
$$

We shall also employ the spin basis $\left(o^{A}, \iota^{A}\right)$ associated with the null tetrad,

$$
l^{a}=o^{A} \bar{o}^{A^{\prime}}, \quad n^{a}=\iota^{A} \bar{\iota}^{A^{\prime}}, \quad m^{a}=o^{A} \bar{\iota}^{A^{\prime}},
$$

normalized by $o_{A} \iota^{A}=1$. Note that this coordinate and tetrad system has some more gauge freedom associated with it. In particular we may make another choice $\hat{\Omega}$ with $\hat{\Omega}=\Theta \Omega$ where $\Theta$ is also periodic and takes the value one at $\mathcal{I}^{-}$. Thus

$$
\tilde{g}_{a b}=\hat{\Omega}^{-2} \hat{g}_{a b}=\Omega^{-2} g_{a b},
$$

and so $\hat{g}_{a b}=\Theta^{2} g_{a b}$. We assume that $\Theta=1+f\left(v, r, x^{I}\right)$ with $f=O(r)$. This will change the definition of the affine parameter $r$, to $\hat{r}$ say, and then we must accompany the change of conformal factor with a null-rotation of the tetrad so that $\hat{\delta}$ is tangent to the sphere $\mathcal{S}_{v \hat{r}}$, thus

$$
\begin{aligned}
& \hat{n}_{a}=n_{a}, \\
& \hat{m}_{a}=\Theta\left(m_{a}+Z n_{a}\right), \\
& \hat{l}_{a}=\Theta^{2}\left(l_{a}+Z \bar{m}_{a}+\bar{Z} m_{a}+Z \bar{Z} n_{a}\right),
\end{aligned}
$$

where $Z$, which parametrises the null rotation, is fixed by requiring $\hat{\delta} \hat{r}=0$; the associated operators change according to 


$$
\begin{aligned}
& \hat{\Delta}=\Theta^{-2} \Delta, \\
& \hat{\delta}=\Theta^{-1}(\delta+Z \Delta), \\
& \hat{D}=D+Z \bar{\delta}+\bar{Z} \delta+Z \bar{Z} \Delta .
\end{aligned}
$$

With the coordinate $v$ common to both systems, we define $\hat{r}$ as the affine parameter with

$$
\hat{\Delta} \hat{r}=\Theta^{-2} \Delta \hat{r}=1 .
$$

This can be integrated to give

$$
\hat{r}=\int_{0}^{r} \Theta^{2} d r=r+O\left(r^{2}\right),
$$

and we need

$$
0=\hat{\delta} \hat{r}=\Theta^{-1}(\delta \hat{r}+Z \Delta \hat{r}),
$$

so that

$$
Z=-\Theta^{-2} \delta \hat{r}
$$

which can be calculated from (39). Note that $Z=O\left(r^{2}\right)$. We shall need to exploit this gauge freedom below. Next we examine what special values some of the spin coefficients take due to the above choice of the null tetrad (we calculate for the unhatted system, but the same relations hold in the hatted systems). Acting by the commutators (A2) on the coordinate $v$, we find

$$
\gamma+\bar{\gamma}=\bar{\alpha}+\beta-\bar{\pi}=\nu=\mu-\bar{\mu}=0 .
$$

Furthermore, commutators $[\delta, \Delta] r$ and $[\bar{\delta}, \delta] r$ give

$$
\tau-\bar{\alpha}-\beta=\rho-\bar{\rho}=0 .
$$

Applying the remaining commutators on the variables $v, r$ and $x^{I}$ leads to the "frame equations", i.e. the equations for the metric functions $H, C^{I}$ and $P^{I}$ :

$$
\begin{array}{ll}
\Delta H & =-(\varepsilon+\bar{\varepsilon}), \\
\delta H & =-\kappa, \\
\Delta C^{I} & =-2 \pi P^{I}-2 \bar{\pi} \bar{P}^{I}, \\
\bar{\delta} P^{I}-\delta \bar{P}^{I} & =(\alpha-\bar{\beta}) P^{I}-(\bar{\alpha}-\beta) \bar{P}^{I}, \\
\Delta P^{I} & =-(\mu-\gamma+\bar{\gamma}) P^{I}-\bar{\lambda} \bar{P}^{I}, \\
\delta C^{I}-D P^{I} & =-(\rho+\varepsilon-\bar{\varepsilon}) P^{I}-\sigma \bar{P}^{I} .
\end{array}
$$

Since the generators $\gamma(v)$ of $\mathcal{I}^{-}$are affinely parametrized null geodesics, $D l^{a}=0$ on $\mathcal{I}^{-}$. Comparing this with the general relation

$$
D l^{a}=(\varepsilon+\bar{\varepsilon}) l^{a}-\bar{\kappa} m^{a}-\kappa \bar{m}^{a},
$$

we see that

$$
\varepsilon+\bar{\varepsilon}=\kappa=0 \quad \text { on } \mathcal{I}^{-} .
$$

Next we wish to show that the freedom in choosing the basis $(m, \bar{m})$ of the space tangential to $\mathcal{S}_{v r}$ allows us to set $\gamma=0$. From the definition of $\gamma$ (eq. A1) we have $\gamma-\bar{\gamma}=m^{a} \Delta \bar{m}_{a}$. Under the rotation through $\chi$,

$$
m^{a} \rightarrow e^{i \chi} m^{a},
$$


the quantity $\gamma-\bar{\gamma}$ transforms according to

$$
\gamma-\bar{\gamma} \rightarrow \gamma-\bar{\gamma}+i \Delta \chi
$$

so by solving the equation

$$
\Delta \chi=i(\gamma-\bar{\gamma}),
$$

and regarding (40) we can set

$$
\gamma=0 .
$$

Because the $\Delta$-operator is the derivative with respect to the coordinate $r$, further rotation (45) with an $r$-independent function $\chi$ does not violate the equality (48). The quantity $\varepsilon-\bar{\varepsilon}$ under the rotation (45) transforms according to

$$
\varepsilon-\bar{\varepsilon} \rightarrow \varepsilon-\bar{\varepsilon}+i D \chi .
$$

Solving the equation

$$
D \chi=i(\varepsilon-\bar{\varepsilon})
$$

on $\mathcal{I}^{-}$, where $r=0$, we set $\varepsilon=\bar{\varepsilon}$ which, together with (44), implies

$$
\varepsilon=0 \quad \text { on } \mathcal{I}^{-} \text {. }
$$

To end this section, we exploit the gauge freedom (37) and (38) to achieve a further simplification. From the commutator $[\hat{\delta}, \hat{\Delta}]$ (see (A2) with the values of the spin coefficients fixed above) we calculate

$$
\hat{\mu}=\Theta^{-2}\left(\mu+\Theta^{-1} \Delta \Theta\right),
$$

so that we can set $\hat{\mu}=0$ by choosing

$$
\Theta=\exp \left(-\int_{0}^{r} \mu \mathrm{d} r\right) .
$$

Having done this, we omit the hats.

In order to elucidate the differences between our choice of the coordinate system and the null tetrad and those used by Gibbons and Stewart, we conclude this section by giving the details of their construction. Instead of the affine parameter $r$ they use coordinate $u$, defined as follows. Let $\mathcal{S}_{0}^{\prime}$ be a spacelike cut on $\mathcal{I}^{-}$and $\mathcal{N}^{\prime}, \mathcal{S}_{0}^{\prime} \subset \mathcal{N}^{\prime}$, the null hypersurface such that the null generators of $\mathcal{N}^{\prime}$ are orthogonal to $\mathcal{S}_{0}^{\prime}$. Now, the real function $u$ on $\mathcal{N}^{\prime}$ is defined in such a way, that $u=0$ on $\mathcal{S}_{0}^{\prime}$, and $u=$ constant on spacelike two-surfaces $\mathcal{S}_{u}$. The cut $\mathcal{S}_{u}$ defines another null hypersurface $\mathcal{N}_{u}$ with null generators orthogonal to $\mathcal{S}_{u}$. The coordinate $u$ is obtained by setting $u=$ constant on $\mathcal{N}_{u}$. Similarly, the family of null hypersurfaces $\mathcal{N}_{v}^{\prime}$ orthogonal to spacelike cuts $\mathcal{S}_{v}^{\prime}$ on $\mathcal{I}^{-}$, with $v$ being the affine parameter along the null generators of $\mathcal{I}^{-}$, is constructed. Coordinates $x^{I}$ are chosen freely on $\mathcal{S}_{0}^{\prime}$ and propagated into the spacetime along $\mathcal{N}^{\prime}$ and $\mathcal{N}_{u}$. The functions $x^{\mu}=\left(u, v, x^{2}, x^{3}\right)$ constitute a coordinate system in the neighbourhood of $\mathcal{I}^{-}$but note that in these coordinates the vector field $\partial_{v}$ is null, which it is not in our coordinates.

The NP tetrad used in [10] consists of vectors $l$, tangent to $\mathcal{N}_{u}, n$, tangent to $\mathcal{N}_{v}^{\prime}$, and $m, \bar{m}$ spanning the tangent space of $\mathcal{S}_{0}^{\prime}$ and propagated into the space-time. The coordinate expression of the tetrad reads (this should be compared with our expressions (24), (25) and (28)) 


$$
l=Q \partial_{v}, \quad n=\partial_{u}+C^{I} \partial_{I}, \quad m=P^{I} \partial_{I},
$$

where $Q, C^{I}$ and $P^{I}$ are metric functions. In this tetrad, the following equation holds:

$$
\Delta n^{a}=-(\gamma+\bar{\gamma}) n^{a}
$$

Therefore, the null generators of $\mathcal{N}_{v}^{\prime}$ are geodesics, but $u$ is not an affine parameter.

The periodicity of the spacetime is defined as the periodicity of all geometrical quantities in the variable $v$. It is shown in [10] that $K=\partial_{v}$ is the Killing vector of the metric and concluded that the spacetime is stationary. However, $K$ is null everywhere by construction as it is tangent to the null generators of $\mathcal{N}_{u}$, while the stationarity requires the timelike Killing vector. Thus, it is impossible to conclude that the spacetime is stationary from the fact that $K$ is the Killing vector. As was mentioned in the introduction, even the Minkowski spacetime does not possess the Killing vector which is everywhere null and tangent to $\mathcal{I}^{-}$.

In the following, we use the coordinates and the tetrad introduced in the beginning of this section. We show that $K=\partial_{v}$ is a space-time Killing vector which is null on $\mathcal{I}^{-}$, but timelike in its neighbourhood.

\section{Proof of the theorem}

Having chosen coordinates and tetrad and fixed special values of some of the NP coefficients we now analyse all geometric quantities assuming analyticity in the chosen coordinates and periodicity on $\mathcal{I}^{-}$in $v$. Following [10] we introduce the notation

$$
\begin{aligned}
& S_{0}=D \Omega, S_{1}=\delta \Omega, S_{2}=\Delta \Omega, \\
& F=\frac{1}{\Omega}\left(S_{0} S_{2}-S_{1} \bar{S}_{1}\right), \psi_{n}=\frac{\Psi_{n}}{\Omega}, n=0,1,2,3,4,
\end{aligned}
$$

where $\Psi_{n}$ are the NP components of the Weyl spinor (see eq. (A10)). In the case of asymptotically-flat space-time they vanish on $\mathcal{I}^{-}$, so assuming smoothness, the $\psi_{n}$ are regular there. Tangential derivatives of the conformal factor vanish on $\mathcal{I}^{-}$, i.e. $S_{0}=S_{1}=0$, and so, again by smoothness, the quantity $F$ is regular on $\mathcal{I}^{-}$. The remaining component of $\nabla \Omega$ is $S_{2}$ which is 1 on $\mathcal{I}^{-}$(cf. (26)), so that its tangential derivatives also vanish on $\mathcal{I}^{-}$. Equations (17) and (19) are explicitly written down in the NP formalism in Appendix B as (B2a) - (B4c). Equations (B2d)-(B2j) show that on $\mathcal{I}^{-}$

$$
\begin{aligned}
& \sigma \quad=0, \\
& F \quad=0, \\
& \rho \quad=0 \\
& \bar{\pi}=0=\beta+\bar{\alpha}=\tau, \\
& \Delta S_{0}=0, \\
& \Delta S_{2}=0, \\
& \Delta S_{1}=0 .
\end{aligned}
$$


Since $F=0$ on $\mathcal{I}^{-}$, also the tangential derivatives $D F$ and $\delta F$ vanish there. From equations (B4a) and (B4b) we thus obtain

$$
\Phi_{00}=\Phi_{01}=0 \quad \text { on } \mathcal{I}^{-} .
$$

The metric functions $P^{I}$ on $\mathcal{I}^{-}$are given by (29). Inserting this expression into the frame equation $(42 d)$ and using relation (54d) we find

$$
\alpha=-\beta=-\frac{1}{2 \sqrt{2}} \cot \theta \text { on } \mathcal{I}^{-} .
$$

The Ricci identity (A20q) now shows that

$$
\Lambda+\Phi_{11}=\frac{1}{2} \text { on } \mathcal{I}^{-} \text {. }
$$

In order to discover the behaviour of the other relevant quantities we shall take into account the properties of the Bondi mass. In a general asymptotically flat electrovacuum space-time the total mass-energy at $\mathcal{I}^{+}$is defined by the formula (see e.g. [4])

$$
M_{B}=-\frac{1}{2 \sqrt{\pi}} \int \mathrm{d} S\left(\tilde{\Psi}_{2}^{0}+\tilde{\sigma}^{0} \dot{\tilde{\sigma}}^{0}\right) .
$$

By the superscript 0 we denote the leading term in the asymptotic expansion of a quantity, superscripts $1,2, \ldots$ then denote higher-order terms, for example, $\tilde{\sigma}=$ $\tilde{\sigma}^{0} \Omega^{2}+\tilde{\sigma}^{1} \Omega^{3}+\mathcal{O}\left(\Omega^{4}\right)$. The rate of decrease of the Bondi mass is given by

$$
\dot{M}_{B}=-\frac{1}{2 \sqrt{\pi}} \int \mathrm{d} S\left(\dot{\tilde{\sigma}}^{0} \dot{\tilde{\sigma}}^{0}+\tilde{\phi}_{2}^{0} \overline{\tilde{\phi}}_{2}^{0}\right) .
$$

The quantities $\sigma$ and $\phi_{i}, i=0,1,2$, are defined in (A1) and (A26). Following the "conversion table" between $\mathcal{I}^{+}$and $\mathcal{I}^{-}$(see (A14)), we analogously define the Bondi mass at $\mathcal{I}^{-}$by

$$
M_{B}=-\frac{1}{2 \sqrt{\pi}} \int \mathrm{d} S\left(\tilde{\Psi}_{2}^{0}+\tilde{\lambda}^{0} \dot{\tilde{\tilde{\lambda}}}^{0}\right) .
$$

Since radiation comes into the physical space-time through $\mathcal{I}^{-}$but can't exit through it, the total mass energy at $\mathcal{I}^{-}$cannot decrease. Its rate of change in (advanced) time $v$ along $\mathcal{I}^{-}$is given by

$$
\dot{M}_{B}=\frac{1}{2 \sqrt{\pi}} \int \mathrm{d} S\left(\dot{\tilde{\tilde{\lambda}}}^{0} \dot{\tilde{\tilde{\lambda}}}^{0}+\tilde{\phi}_{0}^{0} \overline{\tilde{\phi}}_{0}^{0}\right) .
$$

Now we assume periodicity. But a non-decreasing periodic function must be a constant. Hence, our assumption of periodicity of the mass-energy at $\mathcal{I}^{-}$requires

$$
\dot{\tilde{\lambda}}^{0}=0, \quad \tilde{\phi}_{0}^{0}=0
$$

The leading term in the asymptotic expansion of $\tilde{\Psi}_{0}$ is then $\tilde{\Psi}_{0}^{0}=\ddot{\tilde{\bar{\lambda}}}^{0}=0$. Regarding equations (A16) and (A18) and putting $\tilde{\Psi}_{0}^{0}=0$, we can write the asymptotic expansion of $\Psi_{0}$ near $\mathcal{I}^{-}$as

$$
\Psi_{0}=\Psi_{0}^{1} \Omega^{2}+\mathcal{O}\left(\Omega^{3}\right),
$$


or (cf. eq. (53))

$$
\psi_{0}=\mathcal{O}(\Omega) .
$$

Equation (63) implies

$$
\Delta \Psi_{0}=0 \quad \text { on } \mathcal{I}^{-} .
$$

Similarly, eq. (A27), where we put $\tilde{\phi}_{0}^{0}=0$, implies $\phi_{0} \in \mathcal{O}(\Omega)$ and

$$
\Delta \phi_{0}=\phi_{0}^{1} S_{2} \quad \text { on } \mathcal{I}^{-} \text {. }
$$

The geometrical quantities consist of the tetrad components, which give the metric functions, the spin coefficients and the components of the Weyl and the Ricci tensor on $\mathcal{I}^{-}$. Because of our assumption of the periodicity of gravitational field, the geometrical quantities are all assumed to be periodic in the variable $v$ on $\mathcal{I}^{-}$. We do not assume the periodicity of the electromagnetic field since this field may not have the same symmetries as the gravitational field (this is the issue of inheritance which we shall return to). We have shown that the following spin coefficients vanish on $\mathcal{I}^{-}$(and thus do not depend on $v$ ):

$$
\mu, \rho, \sigma, \kappa, \varepsilon, \nu, \gamma, \pi, \tau \text {. }
$$

The spin coefficients $\alpha$ and $\beta$ are $v$-independent because of (56). Now we wish to show that also the last spin coefficient $\lambda$ is independent of $v$. The Bianchi identity (A23a) together with (65) and (55) shows that

$$
D \Phi_{02}=0 \quad \text { on } \mathcal{I}^{-} \text {. }
$$

If we now apply $D$ to the Ricci identity (A20 $g$ ), we get

$$
D^{2} \lambda=0 \quad \text { on } \mathcal{I}^{-} .
$$

The general solution of this equation on $\mathcal{I}^{-}$is

$$
\lambda=\lambda^{(0)}+v \lambda^{(1)},
$$

where $\lambda^{(0)}$ and $\lambda^{(1)}$ are functions independent of $v$. Since $\lambda$ is assumed to be periodic and a polynomial in $v$ can be periodic only if it is constant, we get $\lambda=\lambda^{(0)}$ and

$$
D \lambda=0 \quad \text { on } \mathcal{I}^{-} .
$$

(we borrow this style of argument from [10] where it is used extensively). The Ricci identity (A20g) then implies

$$
\Phi_{02}=0 \quad \text { on } \mathcal{I}^{-} \text {. }
$$

The Ricci identity $(\mathrm{A} 20 h)$ on $\mathcal{I}^{-}$becomes

$$
\Lambda=0,
$$

and then by (57) $\Phi_{11}=1 / 2$ there. Now from (A22c) and $D$ on (A20k), $D \Phi_{12}$ and $D \Phi_{22}$ vanish at $\mathcal{I}^{-}$. We collect these results and some similar ones as a lemma:

Lemma 4.1. The following are zero on $\mathcal{I}^{-}$

$$
\begin{aligned}
& H, C^{A}, \rho, \sigma, \pi, \kappa, \epsilon, S_{0}, S_{1}, F, \psi_{0}, \Phi_{00}, \Phi_{01}, \Phi_{02}, \phi_{0}, \Lambda, \\
& D P^{A}, D \alpha, D \beta, D S_{2}, D \lambda, D \Phi_{11}, D \Phi_{12}, D \Phi_{22}, D \psi_{1}, D \psi_{2}, D \psi_{3}, D \psi_{4}, D \phi_{1}, D \phi_{2}, \\
& D \Delta S_{0}, D \Delta S_{1}, D \Delta S_{2} .
\end{aligned}
$$


Proof. The first line is done already, as is the second line up to $D \psi_{1}$, which comes from (B5a). From $D$ applied to (B5b)-(B5d) we obtain $D^{2} \psi_{i}=0$ whence by periodicity $D \psi_{i}=0$ at $\mathcal{I}^{-}$, in order for $i=2,3,4$. The same procedure applied to (A29a), (A29b) takes care of $D \phi_{1}, D \phi_{2}$. Then the third line follows from $D$ applied to (B2h)-(B2j).

Now we turn to the proof of the Theorem. We set up an induction with the following inductive hypothesis: Suppose inductively that $\partial_{v} \Delta^{j} Q=0$ at $\mathcal{I}^{-}$for

$0 \leq j \leq k$ with $Q$ one of

$$
H, C^{I}, P^{I}, \epsilon, \pi, \lambda, \beta, \alpha, \rho, \sigma, \kappa, F, \psi_{i}, \Phi_{i j}, \phi_{i}, \Lambda
$$

and for $0 \leq j \leq k+1$ with $Q=S_{i}$.

This is easily seen by the Lemma to hold for $k=0$, so we need to deduce it for $j=k+1$ from its truth for $j \leq k$. In this calculation, we use the fact that $\partial_{v}=D$ at $\mathcal{I}^{-}$, and make extensive use of the commutators (A2). Under the inductive hypothesis, the inductive step follows

- for $H, C^{I}, P^{I}$ from $(42 a),(42 c)$ and $(42 e)$;

- for $\epsilon, \pi, \lambda, \beta, \alpha, \rho, \sigma, \kappa$, respectively, from (A20f), (A20i), (A20j), (A20l), (A20o), $(\mathrm{A} 20 n),(\mathrm{A} 20 m)$ and $(\mathrm{A} 20 c)$;

- for $F$ from $(\mathrm{B} 4 c)$;

- for $\phi_{0}$ and $\phi_{1}$ directly from (A29c) and (A29d) respectively; for $\phi_{2}$, from (A29b) we deduce at $\mathcal{I}^{-}$

$$
D^{2} \Delta^{k+1} \phi_{2}=0
$$

and then periodicity implies

$$
D \Delta^{k+1} \phi_{2}=0
$$

- for $\psi_{i}, i=0,1,2,3$ from (B5e)-(B5h); for $\psi_{4}$, under the inductive hypothesis, we deduce at $\mathcal{I}^{-}$

$$
D^{2} \Delta^{k+1} \psi_{4}=0
$$

from $(\mathrm{B} 5 d)$ and then periodicity implies

$$
D \Delta^{k+1} \psi_{4}=0 ;
$$

- for $\Phi_{00}, \Phi_{01}, \Phi_{02}, \Phi_{12}$ from (A22b), (A23b), (A22d) and (A23d) respectively, all with $\Psi_{n}=\Omega \psi_{n}$; then for $\Lambda, \Phi_{11}$ and $\Phi_{22}$ we use (A20h), (A24c) and (A20k).

This completes the inductive step for the first set of quantities $Q$. For $Q=S_{i}$ we use $D \Delta^{k+1}$ applied to (B2h)-(B2j). Thus $r$-derivatives of all orders of the quantities in (74), which includes the metric functions $H, C^{I}$ and $P^{I}$, are independent of $v$. Now analyticity in $r$ forces these functions to be independent of $v$. Therefore, by (30), the metric components are all independent of $v$ and so $K:=\partial / \partial v$ is a Killing vector of the unphysical metric. However, for any $j$,

$$
\partial_{v} \Delta^{j} \Omega=\partial_{v} \Delta^{j-1} S_{2},
$$

at $\mathcal{I}^{-}$and the r.h.s. vanishes for all $j$. Thus, by analyticity in $r, \Omega$ is also independent of $v$ and so $K$ is a Killing vector of the physical metric too. The norm-squared of the Killing vector is

$$
g(K, K)=2(H-\omega \bar{\omega})
$$


This is $O\left(r^{2}\right)$ at $\mathcal{I}^{-}$but there

$$
\Delta^{2} g(K, K)=2 \Delta^{2} H=-2 \Delta(\varepsilon+\bar{\varepsilon})=2
$$

so that $K$ is null at $\mathcal{I}^{-}$but time-like just inside: the metric is stationary.

This completes the proof of the theorem. Note that we have shown that, under the assumption of periodicity of the space-time and the electromagnetic field, both fields are necessarily time-independent(in fact we assume slightly less, namely that the space-time is periodic and that $\phi_{2}$ is periodic). A slightly different question is whether a stationary asymptotically-flat gravitational field might be produced by an electromagnetic field which is not itself stationary. The content of the Corollary 1.2 is that the answer is no.

Proof of Corollary 1.2. Starting from the assumption that the metric admits $\partial_{v}$ as a Killing vector, we want to show that this is also a symmetry of the Maxwell field. We have

$$
\tilde{\Phi}_{i j}=\Omega^{2} \phi_{i} \bar{\phi}_{j}
$$

and $\partial_{v} \tilde{\Phi}_{i j}=0$ so that, for some $\chi$ possibly depending on $v$, we have

$$
\phi_{i}=e^{i \chi} \varphi_{i},
$$

where $\varphi_{i}$ is $v$-independent. From the Maxwell equation (A29a), with $\phi_{0}=0$ on $\mathcal{I}^{-}$, we find $\phi_{1} D \chi=0$ on $\mathcal{I}^{-}$so that $D \chi=0$ unless $\phi_{1}=0$ there. If $\phi_{1}=0$ there, (A29b) gives $D \chi=0$ unless $\phi_{2}=0$, so we can conclude that $D \phi_{i}=0$ on $\mathcal{I}^{-}$. Now we set up an induction to show that $D \Delta^{n} \phi_{i}=0$ on $\mathcal{I}^{-}$for all $n \in \mathbb{N}$ and $i=0,1,2$. The inductive hypothesis will be

$$
(\forall k \leq n)(\forall i \in\{0,1,2\})\left(D \Delta^{k} \phi_{i}=0 \text { on } \mathcal{I}^{-}\right) .
$$

Then by $D \Delta^{n}$ on (A29c) and (A29d) we obtain this for $k=n+1$ and $i=0,1$. For $i=2, D \Delta^{n+1}$ on $(\mathrm{A} 29 b)$ gives

$$
D^{2} \Delta^{n+1} \phi_{2}=0 \quad \text { on } \mathcal{I}^{-},
$$

which integrates to give $\Delta^{n+1} \phi_{2}=a v+b$. This would contribute a $v$-dependent term to $\tilde{\Phi}_{22}$ at $\mathcal{O}\left(\Omega^{2 n+4}\right)$, a contradiction unless $a=0$. Then $D \Delta^{n+1} \phi_{2}=0$ on $\mathcal{I}^{-}$, which completes the induction.

By assumption, the Maxwell field is analytic and so has a convergent power series in $r$ near to $\mathcal{I}^{-}$and we have shown that all coefficients are $v$-independent. Since the spinor dyad is Lie-dragged by the Killing vector, this proves that the Maxwell field is too: in this situation the Maxwell field inherits the symmetry.

\section{Acknowledgments}

P. T. gratefully acknowledges hospitality and financial support from the MittagLeffler Institute, Djursholm, Sweden and the Charles University, Prague, and useful discussions with Gary Gibbons and John Stewart. J.B. also acknowledges the discussions with Gary Gibbons and the partial support from the Grant GA CR 202/09/00772 of the Czech Republic, of Grant No LC06014 and MSM0021620860 of the Ministry of Education. The work of M. S. was supported by the Grant GAUK no. 22708 of the Charles University, Czech Republic. 
No periodic asymptotically flat solutions of the Einstein-Maxwell equations.

\section{Appendix A: The Newman-Penrose formalism and conformal transformations in Einstein-Maxwell space-times}

\section{A1. Gravitational field}

In the NP formalism, the spin coefficients are the Ricci rotation coefficients with respect to a null tetrad $\{l, n, m\}$ with the corresponding spin basis $o_{A}, \iota_{A}$; they encode the connection. The twelve independent complex coefficients are defined by (see e.g. [22], [14] for details)

$$
\begin{aligned}
\kappa & =m^{a} D l_{a}=o^{A} D o_{A}, & \tau & =m^{a} \Delta l_{a}=o^{A} \Delta o_{A}, \\
\sigma & =m^{a} \delta l_{a}=o^{A} \delta o_{A}, & \rho & =m^{a} \bar{\delta} l_{a}=o^{A} \bar{\delta} o_{A}, \\
\varepsilon & =\frac{1}{2}\left[n^{a} D l_{a}-\bar{m}^{a} D m_{a}\right]=\iota^{A} D o_{A}, & \beta & =\frac{1}{2}\left[n^{a} \delta l_{a}-\bar{m}^{a} \delta m_{a}\right]=\iota^{A} \delta o_{A}, \\
\gamma & =\frac{1}{2}\left[n^{a} \Delta l_{a}-\bar{m}^{a} \Delta m_{a}\right]=\iota^{A} \Delta o_{A}, & \alpha & =\frac{1}{2}\left[n^{a} \bar{\delta} l_{a}-\bar{m}^{a} \bar{\delta} m_{a}\right]=\iota^{A} \bar{\delta} o_{A}, \\
\pi & =n^{a} D \bar{m}_{a}=\iota^{A} D \iota_{A}, & & =n^{a} \Delta \bar{m}_{a}=\iota^{A} \Delta \iota_{A}, \\
\lambda & =n^{a} \bar{\delta} \bar{m}_{a}=\iota^{A} \bar{\delta} \iota_{A}, & \mu & =n^{a} \delta \bar{m}_{a}=\iota^{A} \delta \iota_{A},
\end{aligned}
$$

where $D=\nabla_{l}, \Delta=\nabla_{n}, \delta=\nabla_{m}$. Acting on a scalar, the operators $D, \Delta, \delta$ obey the commutation relations:

$$
\begin{aligned}
D \delta-\delta D & =(\bar{\pi}-\bar{\alpha}-\beta) D-\kappa \Delta+(\bar{\rho}-\bar{\varepsilon}+\varepsilon) \delta+\sigma \bar{\delta}, \\
\Delta D-D \Delta & =(\gamma+\bar{\gamma}) D+(\varepsilon+\bar{\varepsilon}) \Delta-(\bar{\tau}+\pi) \delta-(\tau+\bar{\pi}) \bar{\delta}, \\
\Delta \delta-\delta \Delta & =\bar{\nu} D+(\bar{\alpha}+\beta-\tau) \Delta+(\gamma-\bar{\gamma}-\mu) \delta-\bar{\lambda} \bar{\delta}, \\
\delta \bar{\delta}-\bar{\delta} \delta & =(\mu-\bar{\mu}) D+(\rho-\bar{\rho}) \Delta+(\bar{\alpha}-\beta) \bar{\delta}-(\alpha-\bar{\beta}) \delta .
\end{aligned}
$$

The Riemann tensor can be decomposed as follows:

$$
\begin{aligned}
R_{a b c d} & =C_{a b c d} \\
& +\Phi_{A B C^{\prime} D^{\prime}} \epsilon_{A^{\prime} B^{\prime}} \epsilon_{C D}+\bar{\Phi}_{A^{\prime} B^{\prime} C D} \epsilon_{A B} \epsilon_{C^{\prime} D^{\prime}} \\
& +\Lambda\left(\epsilon_{A C} \epsilon_{B D}+\epsilon_{B C} \epsilon_{A D}\right) \epsilon_{A^{\prime} B^{\prime}} \epsilon_{C^{\prime} D^{\prime}} \\
& +\Lambda\left(\epsilon_{A^{\prime} C^{\prime}} \epsilon_{B^{\prime} D^{\prime}}+\epsilon_{B^{\prime} C^{\prime}} \epsilon_{A^{\prime} D^{\prime}}\right) \epsilon_{A B} \epsilon_{C D} .
\end{aligned}
$$

The first part is the Weyl tensor whose spinor equivalent is the totally symmetric Weyl spinor $\Psi_{A B C D}$ :

$$
C_{a b c d}=\Psi_{A B C D} \epsilon_{A^{\prime} B^{\prime}} \epsilon_{C^{\prime} D^{\prime}}+\bar{\Psi}_{A^{\prime} B^{\prime} C^{\prime} D^{\prime}} \epsilon_{A B} \epsilon_{C D} .
$$

The scalar $\Lambda$ is related to the scalar curvature $R$ by

$$
\Lambda=\frac{1}{24} R \text {. }
$$

The symmetric Ricci spinor $\Phi_{A B C^{\prime} D^{\prime}}$ is equivalent to the trace-free part of the Ricci tensor:

$$
R_{a b}=-2 \Phi_{A B A^{\prime} B^{\prime}}+6 \Lambda \epsilon_{A B} \epsilon_{A^{\prime} B^{\prime}} .
$$


The spinor equivalent of the Einstein tensor is

$$
G_{a b}=-2 \Phi_{A B A^{\prime} B^{\prime}}-6 \Lambda \epsilon_{A B} \epsilon_{A^{\prime} B^{\prime}}
$$

and the spinor equivalent of Einstein's equations is

$$
\Phi_{A B A^{\prime} B^{\prime}}=-3 \Lambda \epsilon_{A B} \epsilon_{A^{\prime} B^{\prime}}+4 \pi T_{A B A^{\prime} B^{\prime}} .
$$

Taking the symmetric part or contracting them with $\epsilon^{A B} \epsilon^{A^{\prime} B^{\prime}}$, respectively, we obtain two equations, equivalent to (A8):

$$
\begin{aligned}
& \Phi_{A B A^{\prime} B^{\prime}}=4 \pi T_{(A B)\left(A^{\prime} B^{\prime}\right)}, \\
& 3 \Lambda=\pi T_{A}^{A}{ }_{A^{\prime}}^{A^{\prime}} .
\end{aligned}
$$

The five complex components of the Weyl spinor are

$$
\begin{aligned}
& \Psi_{0}=C_{a b c d} l^{a} m^{b} l^{c} m^{d}=\Psi_{A B C D} o^{A} o^{B} o^{C} o^{D}, \\
& \Psi_{1}=C_{a b c d} l^{a} n^{b} l^{c} m^{d}=\Psi_{A B C D} o^{A} o^{B} o^{C} \iota^{D}, \\
& \Psi_{2}=C_{a b c d} l^{a} m^{b} \bar{m}^{c} n^{d}=\Psi_{A B C D} o^{A} O^{B} \iota^{C} \iota^{D}, \\
& \Psi_{3}=C_{a b c d} l^{a} n^{b} \bar{m}^{c} n^{d}=\Psi_{A B C D} o^{A} \iota^{B} \iota^{C} \iota^{D}, \\
& \Psi_{4}=C_{a b c d} \bar{m}^{a} n^{b} \bar{m}^{c} n^{d}=\Psi_{A B C D} \iota^{A} \iota^{B} \iota^{C} \iota^{D} .
\end{aligned}
$$

The traceless Ricci tensor has the following components (3 real and 3 complex):

$$
\begin{aligned}
& \Phi_{00}=-\frac{1}{2} R_{a b} l^{a} l^{b} \quad=\Phi_{A B A^{\prime} B^{\prime}} o^{A} o^{B} \bar{o}^{A^{\prime}} \bar{o}^{B^{\prime}}, \\
& \Phi_{01}=-\frac{1}{2} R_{a b} l^{a} m^{b} \quad=\Phi_{A B A^{\prime} B^{\prime}} O^{A} o^{B} \bar{o}^{A^{\prime}} \bar{\iota}^{B^{\prime}}, \\
& \Phi_{02}=-\frac{1}{2} R_{a b} m^{a} m^{b} \quad=\Phi_{A B A^{\prime} B^{\prime}} O^{A} o^{B} \bar{\iota}^{A^{\prime}} \bar{\iota}^{B^{\prime}}, \\
& \Phi_{11}=-\frac{1}{4} R_{a b}\left(l^{a} n^{b}+m^{a} \bar{m}^{b}\right)=\Phi_{A B A^{\prime} B^{\prime}} O^{A} \iota^{B} \bar{o}^{A^{\prime}} \bar{\iota}^{B^{\prime}}, \\
& \Phi_{12}=-\frac{1}{2} R_{a b} n^{a} m^{b} \quad=\Phi_{A B A^{\prime} B^{\prime}} O^{A} \iota^{B} \bar{\iota}^{A^{\prime}} \bar{\iota}^{B^{\prime}}, \\
& \Phi_{22}=-\frac{1}{2} R_{a b} n^{a} n^{b} \quad=\Phi_{A B A^{\prime} B^{\prime}} \iota^{A} \iota^{B} \bar{\iota}^{A^{\prime}} \bar{\iota}^{B^{\prime}} .
\end{aligned}
$$

The three remaining components can be obtained via the condition $\Phi_{i j}=\bar{\Phi}_{j i}$. Under the conformal rescaling $g_{a b}=\Omega^{2} \tilde{g}_{a b}$ the covariant derivative acting on a 2-component spinor transforms as

$$
\tilde{\nabla}_{A A^{\prime}} \xi_{B}=\nabla_{A A^{\prime}} \xi_{B}+\Omega^{-1} \xi_{A} \nabla_{B A^{\prime}} \Omega
$$

The NP quantities also transform. To find relations between the physical and unphysical quantities we have to transform the null tetrad. We wish to keep $n_{a}=\tilde{n}_{a}=\partial_{a} v$ so the correct choice is

$$
\begin{array}{llll}
o^{A}=\tilde{o}^{A}, & \iota^{A}=\Omega^{-1} \tilde{\iota}^{A}, & o_{A}=\Omega \tilde{o}_{A}, & \iota_{A}=\tilde{\iota}_{A}, \\
l^{a}=\tilde{l}^{a}, & n^{a}=\Omega^{-2} \tilde{n}^{a}, & m^{a}=\Omega^{-1} \tilde{m}^{a}, & \bar{m}^{a}=\Omega^{-1} \overline{\tilde{m}}^{a} \\
l_{a}=\Omega^{2} \tilde{l}_{a}, & n_{a}=\tilde{n}_{a}, & m_{a}=\Omega \tilde{m}_{a}, & \bar{m}_{a}=\Omega \tilde{\tilde{m}}_{a},
\end{array}
$$

from which the transformation of the spin-coefficients can be found.

The geometrical meaning of the spin coefficients depends on the choice of the null tetrad. With our choices, the vector $l$ is pointing into $\mathcal{I}^{+}$, while $n$ is tangent to $\mathcal{I}^{+}$. On $\mathcal{I}^{-}$the role of these vectors is interchanged, $n$ is pointing from $\mathcal{I}^{-}$and $l$ is tangent to it. To convert quantities from $\mathcal{I}^{+}$to $\mathcal{I}^{-}$we have only to switch the spinors $o^{A}$ and 
$\iota^{A}$ (and adjust some signs). The correspondence between the quantities on $\mathcal{I}^{+}$and $\mathcal{I}^{-}$is given in the following table:

$$
\begin{array}{ll}
\kappa \leftrightarrow \nu, & \tau \leftrightarrow \pi, \\
\sigma \leftrightarrow \lambda, & \rho \leftrightarrow \mu, \\
\varepsilon \leftrightarrow \gamma, & \alpha \leftrightarrow \beta, \\
\Psi_{n} \leftrightarrow \Psi_{4-n}, & \Phi_{i j} \leftrightarrow \Phi_{(2-i)(2-j)}
\end{array}
$$

The scalar curvature and the Ricci spinor transform according to the formulas

$$
\begin{aligned}
& \tilde{R}=\Omega^{2} R-6 \Omega \square \Omega+12 g^{a b}\left(\nabla_{a} \Omega\right)\left(\nabla_{b} \Omega\right), \\
& \tilde{\Phi}_{A B A^{\prime} B^{\prime}}=\Phi_{A B A^{\prime} B^{\prime}}+\Omega^{-1} \nabla_{A\left(A^{\prime}\right.} \nabla_{\left.B^{\prime}\right) B} \Omega,
\end{aligned}
$$

the NP components of the Weyl spinor as

$$
\tilde{\Psi}_{n}=\Omega^{n} \Psi_{n} .
$$

The Weyl spinor is conformally invariant with weight zero:

$$
\Psi_{A B C D}=\tilde{\Psi}_{A B C D} .
$$

Because the physical Weyl spinor vanishes on $\mathcal{I}^{-}$, so does the unphysical one, and assuming smoothness is therefore $\mathcal{O}(\Omega)$. Then we get

$$
\tilde{\Psi}_{n} \in \mathcal{O}\left(\Omega^{n+1}\right) .
$$

The Ricci identities can be written in the spinor form as follows:

$$
\begin{aligned}
& \nabla_{A^{\prime}(A} \nabla_{B)}^{A^{\prime}} \xi_{C}=\Psi_{A B C D} \xi^{D}-2 \Lambda \xi_{(A} \epsilon_{B) C}, \\
& \nabla_{A\left(A^{\prime}\right.} \nabla_{\left.B^{\prime}\right)}^{A} \xi_{C}=\Phi_{C D A^{\prime} B^{\prime}} \xi^{D} .
\end{aligned}
$$

Substituting the basis spinors $o_{A}$ and $\iota_{A}$ for $\xi_{A}$ and projecting the last equations onto the spin basis we obtain the Ricci identities in the NP-formalism:

$$
\begin{aligned}
& D \rho-\bar{\delta} \kappa=\rho^{2}+(\epsilon+\bar{\epsilon}) \rho-\kappa(3 \alpha+\bar{\beta}-\pi)-\tau \bar{\kappa}+\sigma \bar{\sigma}+\Phi_{00} \\
& D \sigma-\delta \kappa=(\rho+\bar{\rho}+3 \varepsilon-\bar{\varepsilon}) \sigma-(\tau-\bar{\pi}+\bar{\alpha}+3 \beta) \kappa+\Psi_{0} \\
& D \tau-\Delta \kappa=\rho(\tau+\bar{\pi})+\sigma(\bar{\tau}+\pi)+(\varepsilon-\bar{\varepsilon}) \tau-(3 \gamma+\bar{\gamma}) \kappa+\Psi_{1}+\Phi_{01} \\
& D \alpha-\bar{\delta} \varepsilon=(\rho+\bar{\varepsilon}-2 \varepsilon) \alpha+\beta \bar{\sigma}-\bar{\beta} \varepsilon-\kappa \lambda-\bar{\kappa} \gamma+(\varepsilon+\rho) \pi+\Phi_{10} \\
& D \beta-\delta \varepsilon=(\alpha+\pi) \sigma+(\bar{\rho}-\bar{\varepsilon}) \beta-(\mu+\gamma) \kappa-(\bar{\alpha}-\bar{\pi}) \varepsilon+\Psi_{1} \\
& D \gamma-\Delta \varepsilon=(\tau+\bar{\pi}) \alpha+(\bar{\tau}+\pi) \beta-(\varepsilon+\bar{\varepsilon}) \gamma-(\gamma+\bar{\gamma}) \varepsilon+\tau \pi-\nu \kappa \\
& \quad+\Psi_{2}-\Lambda+\Phi_{11} \\
& D \lambda-\bar{\delta} \pi=(\rho-3 \varepsilon+\bar{\varepsilon}) \lambda+\bar{\sigma} \mu+(\pi+\alpha-\bar{\beta}) \pi-\nu \bar{\kappa}+\Phi_{20} \\
& D \mu-\delta \pi=(\bar{\rho}-\varepsilon-\bar{\varepsilon}) \mu+\sigma \lambda+(\bar{\pi}-\bar{\alpha}+\beta) \pi-\nu \kappa+\Psi_{2}+2 \Lambda \\
& D \nu-\Delta \pi=(\pi+\bar{\tau}) \mu+(\bar{\pi}+\tau) \lambda+(\gamma-\bar{\gamma}) \pi-(3 \varepsilon+\bar{\varepsilon}) \nu+\Psi_{3}+\Phi_{21} \\
& \Delta \lambda-\bar{\delta} \nu=-(\mu+\bar{\mu}+3 \gamma-\bar{\gamma}) \lambda+(3 \alpha+\bar{\beta}+\pi-\bar{\tau}) \nu-\Psi_{4} \\
& \Delta \mu-\delta \nu=-(\mu+\gamma+\bar{\gamma}) \mu-\lambda \bar{\lambda}+\bar{\nu} \pi+(\bar{\alpha}+3 \beta-\tau) \nu-\Phi_{22} \\
& \Delta \beta-\delta \gamma=(\bar{\alpha}+\beta-\tau) \gamma-\mu \tau+\sigma \nu+\varepsilon \bar{\nu}+(\gamma-\bar{\gamma}-\mu) \beta-\alpha \bar{\lambda}-\Phi_{12} \\
& \Delta \sigma-\delta \tau=-(\mu-3 \gamma+\bar{\gamma}) \sigma-\bar{\lambda} \rho-(\tau+\beta-\bar{\alpha}) \tau+\kappa \bar{\nu}-\Phi_{02} \\
& \Delta \rho-\bar{\delta} \tau=(\gamma+\bar{\gamma}-\bar{\mu}) \rho-\sigma \lambda+(\bar{\beta}-\alpha-\bar{\tau}) \tau+\nu \kappa-\Psi_{2}-2 \Lambda \\
& \Delta \alpha-\bar{\delta} \gamma=(\rho+\varepsilon) \nu-(\tau+\beta) \lambda+(\bar{\gamma}-\bar{\mu}) \alpha+(\bar{\beta}-\bar{\tau}) \gamma-\Psi_{3} \\
& \delta \rho-\bar{\delta} \sigma=(\bar{\alpha}+\beta) \rho-(3 \alpha-\bar{\beta}) \sigma+(\rho-\bar{\rho}) \tau+(\mu-\bar{\mu}) \kappa-\Psi_{1}+\Phi_{01} \\
& \delta \alpha-\bar{\delta} \beta=\mu \rho-\lambda \sigma+\alpha \bar{\alpha}+\beta \bar{\beta}-2 \alpha \beta+(\rho-\bar{\rho}) \gamma+(\mu-\bar{\mu}) \varepsilon-\Psi_{2}+\Lambda+\Phi_{11}, \\
& \delta \lambda-\bar{\delta} \mu=(\rho-\bar{\rho}) \nu+(\mu-\bar{\mu}) \pi+(\alpha+\bar{\beta}) \mu+(\bar{\alpha}-3 \beta) \lambda-\Psi_{3}+\Phi_{21}
\end{aligned}
$$


No periodic asymptotically flat solutions of the Einstein-Maxwell equations.

The spinor form of the Bianchi identities is

$$
\nabla_{B^{\prime}}^{D} \Psi_{A B C D}=\nabla_{A}^{A^{\prime}} \Phi_{B C A^{\prime} B^{\prime}}+\epsilon_{C(A} \nabla_{B) B^{\prime}} \Lambda-\frac{3}{2} \epsilon_{A B} \nabla_{C B^{\prime}} \Lambda
$$

Projecting these equations onto the spin basis leads to the Bianchi identities in the NP formalism:

$$
\begin{aligned}
& D \Psi_{1}-\bar{\delta} \Psi_{0}-D \Phi_{01}+\delta \Phi_{00}=(\pi-4 \alpha) \Psi_{0}+2(2 \rho+\varepsilon) \Psi_{1}-3 \kappa \Psi_{2}+2 \kappa \Phi_{11} \\
& -(\bar{\pi}-2 \bar{\alpha}-2 \beta) \Phi_{00}-2 \sigma \Phi_{10}-2(\bar{\rho}+\varepsilon) \Phi_{01}+\bar{\kappa} \Phi_{02}, \\
& D \Psi_{2}-\bar{\delta} \Psi_{1}+\Delta \Phi_{00}-\bar{\delta} \Phi_{01}+2 D \Lambda=-\lambda \Psi_{0}+2(\pi-\alpha) \Psi_{1}+3 \rho \Psi_{2}-2 \kappa \Psi_{3} \\
& +2 \rho \Phi_{11}+\bar{\sigma} \Phi_{02}+(2 \gamma+2 \bar{\gamma}-\bar{\mu}) \Phi_{00}-2(\alpha+\bar{\tau}) \Phi_{01}-2 \tau \Phi_{10}, \\
& D \Psi_{3}-\bar{\delta} \Psi_{2}-D \Phi_{21}+\delta \Phi_{20}-2 \bar{\delta} \Lambda=-2 \lambda \Psi_{1}+3 \pi \Psi_{2}+2(\rho-\varepsilon) \Psi_{3}-\kappa \Psi_{4} \\
& +2 \mu \Phi_{10}-2 \pi \Phi_{11}-(2 \beta+\bar{\pi}-2 \bar{\alpha}) \Phi_{20}-2(\bar{\rho}-\varepsilon) \Phi_{21}+\bar{\kappa} \Phi_{22}, \\
& D \Psi_{4}-\bar{\delta} \Psi_{3}+\Delta \Phi_{20}-\bar{\delta} \Phi_{21}=-3 \lambda \Psi_{2}+2(\alpha+2 \pi) \Psi_{3}+(\rho-4 \varepsilon) \Psi_{4}+2 \nu \Phi_{10} \\
& -2 \lambda \Phi_{11}-(2 \gamma-2 \bar{\gamma}+\bar{\mu}) \Phi_{20}-2(\bar{\tau}-\alpha) \Phi_{21}+\bar{\sigma} \Phi_{22}, \\
& \Delta \Psi_{0}-\delta \Psi_{1}+D \Phi_{02}-\delta \Phi_{01}=(4 \gamma-\mu) \Psi_{0}-2(2 \tau+\beta) \Psi_{1}+3 \sigma \Psi_{2} \\
& +(\bar{\rho}+2 \varepsilon-2 \bar{\varepsilon}) \Phi_{02}+2 \sigma \Phi_{11}-2 \kappa \Phi_{12}-\bar{\lambda} \Phi_{00}+2(\bar{\pi}-\beta) \Phi_{01}, \\
& \Delta \Psi_{1}-\delta \Psi_{2}-\Delta \Phi_{01}+\bar{\delta} \Phi_{02}-2 \delta \Lambda=\nu \Psi_{0}+2(\gamma-\mu) \Psi_{1}-3 \tau \Psi_{2}+2 \sigma \Psi_{3} \\
& -\bar{\nu} \Phi_{00}+2(\bar{\mu}-\gamma) \Phi_{01}+(2 \alpha+\bar{\tau}-2 \bar{\beta}) \Phi_{02}+2 \tau \Phi_{11}-2 \rho \Phi_{12}, \\
& \Delta \Psi_{2}-\delta \Psi_{3}+D \Phi_{22}-\delta \Phi_{21}+2 \Delta \Lambda=2 \nu \Psi_{1}-3 \mu \Psi_{2}+2(\beta-\tau) \Psi_{3}+\sigma \Psi_{4} \\
& -2 \mu \Phi_{11}-\bar{\lambda} \Phi_{20}+2 \pi \Phi_{12}+2(\beta+\bar{\pi}) \Phi_{21}+(\bar{\rho}-2 \varepsilon-2 \bar{\varepsilon}) \Phi_{22}, \\
& \Delta \Psi_{3}-\delta \Psi_{4}-\Delta \Phi_{21}+\bar{\delta} \Phi_{22}=3 \nu \Psi_{2}-2(\gamma+2 \mu) \Psi_{3}+(4 \beta-\tau) \Psi_{4}-2 \nu \Phi_{11} \\
& -\bar{\nu} \Phi_{20}+2 \lambda \Phi_{12}+2(\gamma+\bar{\mu}) \Phi_{21}+(\bar{\tau}-2 \bar{\beta}-2 \alpha) \Phi_{22}, \\
& D \Phi_{11}-\delta \Phi_{10}+\Delta \Phi_{00}-\bar{\delta} \Phi_{01}+3 D \Lambda=(2 \gamma+2 \bar{\gamma}-\mu-\bar{\mu}) \Phi_{00}+(\pi-2 \alpha-2 \bar{\tau}) \Phi_{01} \\
& +(\bar{\pi}-2 \bar{\alpha}-2 \tau) \Phi_{10}+2(\rho+\bar{\rho}) \Phi_{11}+\bar{\sigma} \Phi_{02}+\sigma \Phi_{20}-\bar{\kappa} \Phi_{12}-\kappa \Phi_{21}, \\
& D \Phi_{12}-\delta \Phi_{11}+\Delta \Phi_{01}-\bar{\delta} \Phi_{02}+3 \delta \Lambda=(2 \gamma-\mu-2 \bar{\mu}) \Phi_{01}+\bar{\nu} \Phi_{00}-\bar{\lambda} \Phi_{10} \\
& +2(\bar{\pi}-\tau) \Phi_{11}+(\pi+2 \bar{\beta}-2 \alpha-\bar{\tau}) \Phi_{02}+(2 \rho+\bar{\rho}-2 \bar{\varepsilon}) \Phi_{12}+\sigma \Phi_{21}-\kappa \Phi_{22},
\end{aligned}
$$


No periodic asymptotically flat solutions of the Einstein-Maxwell equations.

\section{A2. Electromagnetic field}

For the description of an electromagnetic field we use the electromagnetic field tensor $F_{a b}$ and its spinor equivalent $\phi_{A B}$ :

$$
F_{a b}=\phi_{A B} \epsilon_{A^{\prime} B^{\prime}}+\bar{\phi}_{A^{\prime} B^{\prime}} \epsilon_{A B} .
$$

The NP components of the Maxwell spinor are defined by

$$
\begin{aligned}
& \phi_{0}=F_{a b} l^{a} m^{b}=\phi_{A B} o^{A} o^{B} \\
& \phi_{1}=\frac{1}{2} F_{a b}\left[l^{a} n^{b}-m^{a} \bar{m}^{b}\right]=\phi_{A B} o^{A} \iota^{B} \\
& \phi_{2}=F_{a b} \bar{m}^{a} n^{b}=\phi_{A B} \iota^{A} \iota^{B}
\end{aligned}
$$

The conformal transformation of these quantities is given by

$$
\tilde{\phi}_{A B}=\Omega \phi_{A B}, \quad \tilde{\phi}_{i}=\Omega^{i+1} \phi_{i} .
$$

Maxwell's equations without sources are equivalent to the (conformally-invariant) spin-1 zero-rest-mass equation

$$
\nabla_{A^{\prime}}^{A} \phi_{A B}=0 .
$$

Projecting this onto the spin basis we obtain Maxwell's equations in the NP formalism:

$$
\begin{aligned}
D \phi_{1}-\bar{\delta} \phi_{0} & =(\pi-2 \alpha) \phi_{0}+2 \rho \phi_{1}-\kappa \phi_{2}, \\
D \phi_{2}-\bar{\delta} \phi_{1} & =-\lambda \phi_{0}+2 \pi \phi_{1}+(\rho-2 \varepsilon) \phi_{2}, \\
\Delta \phi_{0}-\delta \phi_{1} & =(2 \gamma-\mu) \phi_{0}-2 \tau \phi_{1}+\sigma \phi_{2}, \\
\Delta \phi_{1}-\delta \phi_{2} & =\nu \phi_{0}-2 \mu \phi_{1}+(2 \beta-\tau) \phi_{2} .
\end{aligned}
$$

\section{Appendix B: Conformal field equations}

\section{B1. Einstein-Maxwell fields}

The projections of the equation (17),

$\nabla_{A A^{\prime}} \nabla_{B B^{\prime}} \Omega=\Omega^{3} \phi_{A B} \bar{\phi}_{A^{\prime} B^{\prime}}-\Omega \Phi_{A B A^{\prime} B^{\prime}}+(F+\Omega \Lambda) \epsilon_{A^{\prime} B^{\prime}} \epsilon_{A B}$,

onto the null tetrad imply the following system of equations:

$$
\begin{array}{ll}
D S_{0}+(\varepsilon+\bar{\varepsilon}) S_{0}+\bar{\kappa} S_{1}+\kappa \bar{S}_{1} & =\Omega^{3} \phi_{0} \bar{\phi}_{0}-\Omega \Phi_{00} \\
D S_{1}-\bar{\pi} S_{0}+(\bar{\varepsilon}-\varepsilon) S_{1}+\kappa S_{2} & =\Omega^{3} \phi_{0} \bar{\phi}_{1}-\Omega \Phi_{01} \\
\delta S_{0}-(\bar{\alpha}+\beta) S_{0}+\bar{\rho} S_{1}+\sigma \bar{S}_{1} & =\Omega^{3} \phi_{0} \bar{\phi}_{1}-\Omega \Phi_{01} \\
\delta S_{1}-\bar{\lambda} S_{0}+(\bar{\alpha}-\beta) S_{1}+\sigma S_{2} & =\Omega^{3} \phi_{0} \bar{\phi}_{2}-\Omega \Phi_{02} \\
D S_{2}-F-\Omega \Lambda-\pi S_{1}-\bar{\pi} \bar{S}_{1}+(\varepsilon+\bar{\varepsilon}) S_{2} & =\Omega^{3} \phi_{1} \bar{\phi}_{1}-\Omega \Phi_{11} \\
\delta \bar{S}_{1}+F+\Omega \Lambda-\mu S_{0}+(\beta-\bar{\alpha}) \bar{S}_{1}+\bar{\rho} S_{2} & =\Omega^{3} \phi_{1} \bar{\phi}_{1}-\Omega \Phi_{11} \\
\delta S_{2}-\mu S_{1}-\bar{\lambda} \bar{S}_{1}+(\bar{\alpha}+\beta) S_{2} & =\Omega^{3} \phi_{1} \bar{\phi}_{2}-\Omega \Phi_{12} \\
\Delta S_{0}-F-\Omega \Lambda-(\gamma+\bar{\gamma}) S_{0}+\bar{\tau} S_{1}+\tau \bar{S}_{1} & =\Omega^{3} \phi_{1} \bar{\phi}_{1}-\Omega \Phi_{11} \\
\Delta S_{1}-\bar{\nu} S_{0}+(\bar{\gamma}-\gamma) S_{1}+\tau S_{2} & =\Omega^{3} \phi_{1} \bar{\phi}_{2}-\Omega \Phi_{12} \\
\Delta S_{2}-\nu S_{1}-\bar{\nu} \bar{S}_{1}+(\gamma+\bar{\gamma}) S_{2} & =\Omega^{3} \phi_{2} \bar{\phi}_{2}-\Omega \Phi_{22} .
\end{array}
$$


No periodic asymptotically flat solutions of the Einstein-Maxwell equations.

The projections of the equation (19),

$$
\nabla_{A A^{\prime}} F=\Omega^{2} \phi_{A}^{B} \bar{\phi}_{A^{\prime}}^{B^{\prime}} \nabla_{B B^{\prime}} \Omega-\Phi_{A B A^{\prime} B^{\prime}} \nabla^{B B^{\prime}} \Omega+\Lambda \nabla_{A A^{\prime}} \Omega,
$$

give

$$
\begin{aligned}
D F & =-S_{0} \Phi_{11}+S_{1} \Phi_{10}+\bar{S}_{1} \Phi_{01}-S_{2} \Phi_{00} \\
& +\Omega^{2}\left[S_{0} \phi_{1} \bar{\phi}_{1}-S_{1} \phi_{1} \bar{\phi}_{0}-\bar{S}_{1} \phi_{0} \bar{\phi}_{1}+S_{2} \phi_{0} \bar{\phi}_{0}\right]+\Lambda S_{0}, \\
\delta F & =-S_{0} \Phi_{12}+S_{1} \Phi_{11}+\bar{S}_{1} \Phi_{02}-S_{2} \Phi_{01} \\
& +\Omega^{2}\left[S_{0} \phi_{1} \bar{\phi}_{2}-S_{1} \phi_{1} \bar{\phi}_{1}-\bar{S}_{1} \phi_{0} \bar{\phi}_{2}+S_{2} \phi_{0} \bar{\phi}_{1}\right]+\Lambda S_{1}, \\
\Delta F & =-S_{0} \Phi_{22}+S_{1} \Phi_{21}+\bar{S}_{1} \Phi_{12}-S_{2} \Phi_{11} \\
& +\Omega^{2}\left[S_{0} \phi_{2} \bar{\phi}_{2}-S_{1} \phi_{2} \bar{\phi}_{1}-\bar{S}_{1} \phi_{1} \bar{\phi}_{2}+S_{2} \phi_{1} \bar{\phi}_{1}\right]+\Lambda S_{2} .
\end{aligned}
$$

The conformal Bianchi identities (9) for the Einstein-Maxwell field projected onto the spin basis imply the following system:

$$
\begin{aligned}
& D \psi_{1}-\bar{\delta} \psi_{0}=(\pi-4 \alpha) \psi_{0}+2(\varepsilon+2 \rho) \psi_{1}-3 \kappa \psi_{2}-3 S_{1} \phi_{0} \bar{\phi}_{0}+3 S_{0} \phi_{0} \bar{\phi}_{1} \\
& +\Omega\left[2 \sigma \phi_{1} \bar{\phi}_{0}-2 \beta \phi_{0} \bar{\phi}_{0}+2 \varepsilon \phi_{0} \bar{\phi}_{1}-2 \kappa \phi_{1} \bar{\phi}_{1}+\bar{\phi}_{0} \delta \phi_{0}-\bar{\phi}_{1} D \phi_{0}\right], \\
& D \psi_{2}-\bar{\delta} \psi_{1}=-\lambda \psi_{0}+2(\pi-\alpha) \psi_{1}+2 \rho \psi_{2}-2 \kappa \psi_{3}-S_{2} \phi_{0} \bar{\phi}_{0}-2 S_{1} \phi_{1} \bar{\phi}_{0} \\
& +2 S_{0} \phi_{1} \bar{\phi}_{1}+\bar{S}_{1} \phi_{0} \bar{\phi}_{1}+\frac{2}{3} \Omega\left[\bar{\phi}_{0} \delta \phi_{1}-\bar{\phi}_{1} D \phi_{1}-(\gamma+\mu) \phi_{0} \bar{\phi}_{0}\right. \\
& \left.+\tau \phi_{1} \bar{\phi}_{0}+(\alpha+\pi) \phi_{0} \bar{\phi}_{1}+\sigma \phi_{2} \bar{\phi}_{0}-\rho \phi_{1} \bar{\phi}_{1}-\kappa \phi_{2} \bar{\phi}_{1}\right]+\frac{1}{3} \Omega\left[\bar{\phi}_{0} \Delta \phi_{0}-\bar{\phi}_{1} \bar{\delta} \phi_{0}\right], \\
& D \psi_{3}-\bar{\delta} \psi_{2}=-2 \lambda \psi_{1}+3 \pi \psi_{2}+2(\rho-\varepsilon) \psi_{3}-\kappa \psi_{4}-2 S_{2} \phi_{1} \bar{\phi}_{0}-S_{1} \phi_{2} \bar{\phi}_{0}+S_{0} \phi_{2} \bar{\phi}_{1}+2 \bar{S}_{1} \phi_{1} \bar{\phi}_{1} \\
& +\frac{2}{3} \Omega\left[-\nu \phi_{0} \bar{\phi}_{0}-\mu \phi_{1} \bar{\phi}_{0}+\lambda \phi_{0} \bar{\phi}_{1}+(\beta+\tau) \phi_{2} \bar{\phi}_{0}+\pi \phi_{1} \bar{\phi}_{1}-(\varepsilon+\rho) \phi_{2} \bar{\phi}_{1}+\bar{\phi}_{0} \Delta \phi_{1}-\bar{\phi}_{1} \bar{\delta} \phi_{1}\right] \\
& +\frac{1}{3} \Omega\left[\bar{\phi}_{0} \delta \phi_{2}-\bar{\phi}_{1} D \phi_{2}\right] \text {, } \\
& D \psi_{4}-\bar{\delta} \psi_{3}=-3 \lambda \psi_{2}+2(\alpha+2 \pi) \psi_{3}+(\rho-4 \varepsilon) \psi_{4}-3 S_{2} \phi_{2} \bar{\phi}_{0}+3 \bar{S}_{1} \phi_{2} \bar{\phi}_{1} \\
& +\Omega\left[\bar{\phi}_{0} \Delta \phi_{2}-\bar{\phi}_{1} \bar{\delta} \phi_{2}-2 \nu \phi_{1} \bar{\phi}_{0}+2 \gamma \phi_{2} \bar{\phi}_{0}+2 \lambda \phi_{1} \bar{\phi}_{1}-2 \alpha \phi_{2} \bar{\phi}_{1}\right] \\
& \delta \psi_{1}-\Delta \psi_{0}=(\mu-4 \gamma) \psi_{0}+2(\beta+2 \tau) \psi_{1}-3 \sigma \psi_{2}-3 S_{1} \phi_{0} \bar{\phi}_{1}+3 S_{0} \phi_{0} \bar{\phi}_{2} \\
& +\Omega\left[-2 \beta \phi_{0} \bar{\phi}_{1}+2 \sigma \phi_{1} \bar{\phi}_{1}+2 \varepsilon \phi_{0} \bar{\phi}_{2}-2 \kappa \phi_{1} \bar{\phi}_{2}-\bar{\phi}_{2} D \phi_{0}+\bar{\phi}_{1} \delta \phi_{0}\right] \text {, } \\
& \delta \psi_{2}-\Delta \psi_{1}=-\nu \psi_{0}+2(\mu-\gamma) \psi_{1}+3 \tau \psi_{2}-2 \sigma \psi_{3}-S_{2} \phi_{0} \bar{\phi}_{1}-2 S_{1} \phi_{1} \bar{\phi}_{1}+2 S_{0} \phi_{1} \bar{\phi}_{1}+\bar{S}_{1} \phi_{0} \bar{\phi}_{2} \\
& +\frac{2}{3} \Omega\left[-(\gamma+\mu) \phi_{0} \bar{\phi}_{1}+\tau \phi_{1} \bar{\phi}_{1}+\sigma \phi_{2} \bar{\phi}_{1}+(\pi+\alpha) \phi_{0} \bar{\phi}_{2}-\rho \phi_{1} \bar{\phi}_{2}-\kappa \phi_{2} \bar{\phi}_{2}\right. \\
& \left.+\bar{\phi}_{1} \delta \phi_{1}-\bar{\phi}_{2} D \phi_{1}\right]+\frac{1}{3} \Omega\left[\bar{\phi}_{1} \Delta \phi_{0}-\bar{\phi}_{2} \bar{\delta} \phi_{0}\right] \text {, } \\
& \delta \psi_{3}-\Delta \psi_{2}=-2 \nu \psi_{1}+3 \mu \psi_{2}+2(\tau-\beta) \psi_{3}-\sigma \psi_{4}-2 S_{2} \phi_{1} \bar{\phi}_{1}-S_{1} \phi_{2} \bar{\phi}_{1}+S_{0} \phi_{2} \bar{\phi}_{2}+2 \bar{S}_{1} \phi_{1} \bar{\phi}_{2} \\
& +\frac{2}{3} \Omega\left[-\nu \phi_{0} \bar{\phi}_{1}-\mu \phi_{1} \bar{\phi}_{1}+(\beta+\tau) \phi_{2} \bar{\phi}_{1}+\lambda \phi_{0} \bar{\phi}_{2}+\pi \phi_{1} \bar{\phi}_{2}-(\varepsilon+\rho) \phi_{2} \bar{\phi}_{2}+\bar{\phi}_{1} \Delta \phi_{1}-\bar{\phi}_{2} \bar{\delta} \phi_{1}\right] \\
& +\frac{1}{3} \Omega\left[\bar{\phi}_{1} \delta \phi_{2}-\bar{\phi}_{2} D \phi_{2}\right] \text {, } \\
& \delta \psi_{4}-\Delta \psi_{3}=-3 \nu \psi_{2}+2(2 \gamma+2 \mu) \psi_{3}+(\tau-4 \beta) \psi_{4}-3 S_{2} \phi_{2} \bar{\phi}_{1}+3 \bar{S}_{1} \phi_{1} \bar{\phi}_{2} \\
& +\Omega\left[-2 \nu \phi_{1} \bar{\phi}_{1}+2 \gamma \phi_{2} \bar{\phi}_{1}+2 \lambda \phi_{1} \bar{\phi}_{2}-2 \alpha \phi_{2} \bar{\phi}_{2}+\bar{\phi}_{1} \Delta \phi_{2}-\bar{\phi}_{2} \bar{\delta} \phi_{2}\right] \text {. }
\end{aligned}
$$




\section{Appendix C: Reissner-Nordström space-time}

To justify our choice of gauge and show that the choice made by [10] is too restrictive we shall show here how a simple space-time, namely, the Reissner-Nordström solution, appears in our gauge. The physical metric is

$\mathrm{d} \tilde{s}^{2}=\left(1-\frac{2 m}{\tilde{r}}+\frac{Q^{2}}{\tilde{r}^{2}}\right) \mathrm{d} t^{2}-\left(1-\frac{2 m}{\tilde{r}}+\frac{Q^{2}}{\tilde{r}^{2}}\right)^{-1} \mathrm{~d} \tilde{r}^{2}-\tilde{r}^{2} \mathrm{~d} \Sigma^{2}$,

where $Q$ is the charge and $\mathrm{d} \Sigma^{2}=\mathrm{d} \theta^{2}+\sin ^{2} \theta \mathrm{d} \phi^{2}$. In the standard conformal compactification of the Reissner-Nordström space-time one introduces the "tortoise coordinate" $r^{*}$ and the advanced time $v$ by

$$
\begin{aligned}
& \mathrm{d} \tilde{r}=\left(1-\frac{2 m}{\tilde{r}}+\frac{Q^{2}}{\tilde{r}^{2}}\right) \mathrm{d} r^{*}, \\
& v=t+r^{*} .
\end{aligned}
$$

In these coordinates the physical metric acquires the form

$$
\mathrm{d} \tilde{s}^{2}=\left(1-\frac{2 m}{\tilde{r}}+\frac{Q^{2}}{\tilde{r}^{2}}\right)\left(\mathrm{d} v^{2}-2 \mathrm{~d} v \mathrm{~d} r^{*}\right)-\tilde{r}^{2} \mathrm{~d} \Sigma^{2} .
$$

We compactify it by defining the coordinate

$$
r=\tilde{r}^{-1}
$$

and the conformal factor

$$
\Omega=r .
$$

The unphysical metric then reads

$$
\mathrm{d} s^{2}=r^{2}\left(1-2 m r+Q^{2} r^{2}\right) \mathrm{d} v^{2}+2 \mathrm{~d} v \mathrm{~d} r-\mathrm{d} \Sigma^{2} .
$$

Comparing this with (31)-(34) we find the metric functions to be

$$
\begin{aligned}
& H=\frac{1}{2} r^{2}-m r^{3}+\frac{1}{2} Q^{2} r^{4}, \\
& C^{I}=0, \\
& P^{2}=\frac{1}{\sqrt{2}}, \\
& P^{3}=\frac{i}{\sqrt{2} \sin \theta} .
\end{aligned}
$$

From the metric the other geometrical quantities follow. The spin coefficients are all zero, except for

$$
\begin{aligned}
& \varepsilon=-\frac{1}{2} r+\frac{3}{2} m r^{2}-Q^{2} r^{3}, \\
& \alpha=-\beta=-\frac{1}{2 \sqrt{2}} \cot \theta .
\end{aligned}
$$

The non-zero components of the Weyl and Ricci tensor read

$$
\begin{aligned}
\psi_{2} & =m-Q^{2} r, \\
\Phi_{11} & =\frac{1}{2}-\frac{3}{2} m r+\frac{3}{2} Q^{2} r^{2}, \\
\Lambda & =\frac{1}{2} m r-\frac{1}{2} Q^{2} r^{2} .
\end{aligned}
$$


The electromagnetic 4-potential and corresponding electromagnetic tensor in these coordinates are

$$
\begin{aligned}
& A_{\mu}=(Q r, 0,0,0), \\
& F_{\mu \nu}=-Q \epsilon_{\mu \nu 23} .
\end{aligned}
$$

The only non-vanishing NP component of $F_{\mu \nu}$ is

$$
\phi_{1}=Q
$$

as one would expect. All these results are in accordance with results obtained in the text. On the other hand, the gauge condition $\Lambda=0$ everywhere, imposed in [10], leads to a periodic unphysical metric only if $m=0$, i.e. flat space-time. This can be seen as follows: we need to rescale the metric (C6) say to

$$
\hat{g}_{a b}=\Theta^{-2} g_{a b}
$$

so that, by (A15),

$$
\hat{\Lambda}=\Theta^{-2}\left(\Theta \Lambda+\frac{1}{4} \square \Theta\right)=0,
$$

where the boundary conditions on $\Theta$ are that $\Theta=1$ on $r=0$ and, say, $v=0$ (in order to preserve the conditions that $\rho=0$ on $r=0, \mu=0$ on $v=0$ and $\Theta=1$ on $v=r=0)$. With the metric (C6) this wave equation on $\Theta$ becomes

$$
2 \partial_{v} \partial_{r} \Theta=\partial_{r}\left(A \partial_{r} \Theta\right)-L^{2} \Theta-2\left(m r-Q r^{2}\right) \Theta,
$$

with $A=r^{2}\left(1-2 m r+Q^{2} r^{2}\right)$ and

$$
L^{2} \Theta=\frac{1}{\sin \theta} \frac{\partial}{\partial \theta}\left(\frac{\partial \Theta}{\partial \theta}\right)+\frac{1}{\sin ^{2} \theta} \frac{\partial^{2} \Theta}{\partial \theta^{2}} .
$$

Now from (C12) evaluated at $r=0$ we calculate $\partial_{v} \partial_{r} \Theta=0$ so that $\partial_{r} \Theta$ is constant on $\mathcal{I}^{-}$, but it vanishes at $v=0$ so it is zero for all $v$. Then from $(\mathrm{C} 12)$ again at $\mathcal{I}^{-}$,

$$
\partial_{v} \partial_{r}^{2} \Theta=-m \text {. }
$$

Thus $\Theta$ cannot be periodic in $v$ unless $m=0$, in which case the physical metric is flat.

[1] Bičák, J. and Schmidt, B. G. 2007 Helical symmetry in linear systems, Phys. Rev. D76, 104040 (11 pages)

[2] Bizoń, P. and Wasserman, A. 2000, On existence of mini-boson stars, Commun. Math. Phys. 215, 357-373.

[3] Bonnor, W. B. Non-radiative solutions of Einstein's equations for dust, Commun. Math. Phys. 51, 191-199.

[4] Exton, A.R., Newman, E.T. and Penrose, R. 1969 Conserved quantities in the Einstein-Maxwell theory J. Math. Phys. 10 1566-1570.

[5] Friedrich, H. 1981 On the regular and the asymptotic characteristic initial value problem for Einstein's vacuum field equations Proc. Roy. Soc. London Ser. A 375 169-184.

[6] Friedrich, H. 1982 On the existence of analytic null asymptotically flat solutions of Einstein's vacuum field equations Proc. Roy. Soc. London Ser. A381 361-371

[7] Friedrich, H., Rácz, I. and Wald, R.M. 1999 On the rigidity theorem for space-times with a stationary event horizon or a compact Cauchy horizon Comm. Math. Phys. 204, 691-707.

[8] Gair, J. R. 2001 Spherical universes with anisotropic pressure Class. Quant. Grav. 18, 48974920.

[9] Galloway, G. J. 1984 Splitting theorems for spatially closed space-times Comm. Math. Phys. 96 $423-429$. 
No periodic asymptotically flat solutions of the Einstein-Maxwell equations.

[10] Gibbons, G. W. and Stewart, J. M. 1984 Absence of asymptotically flat solutions of Einstein's equations which are periodic and empty near infinity in Classical general relativity (London, 1983) 77-94, editors W. Bonnor, J. N. Islam and M. A. H. Callum, Cambridge University Press, Cambridge.

[11] Hawking, S. W. and Ellis, G. F. R. 1973 The large scale structure of space-time Cambridge University Press, London-New York.

[12] Moncrief, V. and Isenberg, J. 1983 Symmetries of cosmological Cauchy horizons. Comm. Math. Phys. 89 387-413.

[13] MacCallum, M.A.H. and Van den Berg, N. Non-inheritance of static symmetry by Maxwell fields, in Galaxies, axisymmetric systems and relativity (Essays presented to W. B. Bonnor in his 65th birthday), edited by M. A. H. McCallum, 138-148, Cambridge University Press, Cambridge-London-New York.

[14] Newman, E.T. and Penrose, R. 1962 An approach to gravitational radiation by a method of spin coefficients J. Math. Phys. 3 566-578.

[15] Papapetrou, A. 1957 Über periodische nichtsinguläre Lösungen in der allgemeinen Relativitätstheorie Ann. Physik (6) 20 399-411.

[16] Papapetrou, A. 1958 Über periodische Gravitations- und elektromagnetische Felder in der allgemeinen Relativitätstheorie Ann. Physik (7) 1 186-197.

[17] Papapetrou, A. 1962 Non-existence of periodically varying non-singular gravitational fields in Les théories relativistes de la gravitation (Royaumont, 1959) pp. 193-198 Editions du Centre National de la Recherche Scientifique, Paris.

[18] Penrose, R. and Rindler, W. 1986 Spinors and space-time vol II Cambridge Monographs on Mathematical Physics. Cambridge University Press, Cambridge.

[19] Schild, A. 1963 Electromagnetic two-body problem Physical Review, vol. 131, Issue 6, pp. 27622766 .

[20] Scholtz, M. and Bičák, J. 2008 On the non-existence of asymptotically flat periodic solutions of Einstein's equations in Week of doctoral students 2008 (June 3 - June 6), Proceeding of contributed papers, Part III-Physics eds. J. Šafránková and J. Pavlů, Matfyzpress, Prague 2008, pp. 179-185.

[21] Stephani, H., Kramer, D., MacCallum, M. A. H., Hoenselaers, C. and Herlt, E. 2003 Exact solutions to Einstein's field equations (Second Edition) Cambridge University Press, Cambridge.

[22] Stewart, J. 1990 Advanced general relativity, Cambridge Monographs on Mathematical Physics. Cambridge University Press, Cambridge,

[23] Tod, P. 2009 On analytic asymptotically-flat vacuum and electrovac metrics, periodic in time arXiv:0902.1061v2[gr-qc], 12 pages.

[24] Trautman, A. 1957 Proof of the non-existence of periodic gravitational fields representing radiation Bull. Acad. Polon. Sci. Cl. III. 5 pp 1115-1117, XCII.

[25] Workshop on Helically Symmetric Systems 2007 Max Planck Institute for Gravitational Physics, Potsdam, Germany, http://www.aei.mpg.de/ workshop/helical/index.html 